\title{
Saccade Modulation by Optical and Electrical Stimulation in the Macaque Frontal Eye Field
}

\author{
Shay Ohayon, ${ }^{1,2}$ Piercesare Grimaldi, ${ }^{1}$ Nicole Schweers, ${ }^{1}$ and Doris Y. Tsao ${ }^{1,2}$ \\ ${ }^{1}$ Division of Biology and ${ }^{2}$ Computation and Neural Systems, California Institute of Technology, Pasadena, California 91125
}

Recent studies have demonstrated that strong neural modulations can be evoked with optogenetic stimulation in macaque motor cortex without observing any evoked movements (Han et al., 2009, 2011; Diester et al., 2011). It remains unclear why such perturbations do not generate movements and if conditions exist under which they may evoke movements. In this study, we examine the effects of five optogenetic constructs in the macaque frontal eye field and use electrical microstimulation to assess whether optical perturbation of the local network leads to observable motor changes during optical, electrical, and combined stimulation. We report a significant increase in the probability of evoking saccadic eye movements when low current electrical stimulation is coupled to optical stimulation compared with when electrical stimulation is used alone. Experiments combining channelrhodopsin 2 (ChR2) and electrical stimulation with simultaneous fMRI revealed no discernible fMRI activity at the electrode tip with optical stimulation but strong activity with electrical stimulation. Our findings suggest that stimulation with current ChR2 optogenetic constructs generates subthreshold activity that contributes to the initiation of movements but, in most cases, is not sufficient to evoke a motor response.

\section{Introduction}

An important step in understanding a cortical circuit is to precisely perturb activity in specific nodes of the circuit. Electrical microstimulation has been used widely in nonhuman primates (NHPs) as a tool to probe perception (Salzman et al., 1990; Murphey and Maunsell, 2007), decision making (Cohen and Newsome, 2004; Moore and Fallah, 2004), motor control (Graziano et al., 2002), and network connectivity (Ekstrom et al., 2008; Moeller et al., 2008; Logothetis et al., 2010). However, the effects of electrical microstimulation and its spread are still poorly understood (Histed et al., 2009), and the technique has several drawbacks, including inability to monitor activity during stimulation attributable to electrical artifacts, inability to target specific subpopulations of neurons, and difficulty in interpreting results because of the possibility of activating fibers of passage. Optogenetics is an emerging technology that can overcome many of these limitations by targeting specific cell populations (Yizhar et al., 2011b) and controlling neural activity to millimeter and millisecond precision (Boyden et al., 2005).

Although optogenetics has been successful in altering and evoking motor movements in rodents (Gradinaru et al., 2007),

Received June 24, 2013; revised Sept. 9, 2013; accepted Sept. 14, 2013.

Author contributions:S.O. and D.Y.T. designed research;S.0. performed research;S.O.,P.G., and N.S. contributed unpublished reagents/analytic tools; S.O. analyzed data; S.O. and D.Y.T. wrote the paper.

This work was supported by National Institute of Health Grant 1R01EY019702, the Della Martin Foundation, and a Searle Scholar Award (D.Y.T.). We thank Sebastian Moeller for his assistance in preparing the electrical microstimulation experiments, David Anderson for using his confocal setup to image histological slices, Ilka Diester for her continuing support with setting up viral injections, Karl Deisseroth for the hSyn-ChR2(H134R)-eYFP, hSynChR2(E123A) - eYFP, CaMKII-ChR2(E123A)-mCherry and hSyn-eNpHR3.0 - eYFP plasmids, and Ed Boyden for the CAG-Arch-eGFP plasmid.

The authors declare no competing financial interests.

Correspondence should be addressed to either Shay Ohayon or Doris Y. Tsao, Division of Biology, California Institute of Technology, 1200 East California Boulevard, Pasadena, CA 91125. E-mail: shay.ohayon@gmail.com, dortsao@caltech.edu.

DOI:10.1523/JNEUROSCI.2675-13.2013

Copyright $\odot 2013$ the authors $\quad 0270-6474 / 13 / 3316684-14 \$ 15.00 / 0$ several attempts in the macaque failed to find any behavioral effects despite strong light-induced modulation of neural activity in premotor cortex (Diester et al., 2011), parietal regions (Han et al., 2011), the frontal eye field (FEF) (Han et al., 2009), primary visual cortex (Ruiz et al., 2013), and subcortical areas involved in motor planning and execution (Galvan et al., 2012). This is surprising because electrical microstimulation in the same areas of the same animals were found to reliably evoke limb (Diester et al., 2011) and eye (E. Boyden, personal communication) movements. Recently, three studies reported observable behavioral changes (Cavanaugh et al., 2012; Gerits et al., 2012; Jazayeri et al., 2012), but only Jazayeri et al. were successful in evoking a movement after optical stimulation [channelrhodopsin 2 (ChR2) in primary visual cortex], suggesting that stimulation evoked a phosphene percept to which monkeys were trained to respond. Although these studies are important in providing evidence that optogenetic perturbations can lead to behavioral changes, they do not address the issue of how effective such perturbations are compared with known methods of perturbing behavior. Furthermore, the puzzle of why robust neural modulation can be evoked in motor areas without any observable induced motor behavior persists.

Various explanations have been proposed to explain the lack of behavioral effects induced by optical stimulation in NHPs, including low cell infection percentage, labeling of cells that do not participate in behavior, small region of stimulated tissue, lack of stimulation of fibers of passage, low stimulation frequencies, and monitoring methods not subtle enough to observe possible effects. In this study, we examined the effects of five optogenetic constructs in the macaque FEF side by side with electrical microstimulation, to assess whether optical perturbation of the local network leads to observable motor changes during optical, electrical, and combined stimulation. Our experiments address these 
Table 1. List of injection sites, their coordinates, and the number of single units recorded

\begin{tabular}{|c|c|c|c|c|c|c|c|c|c|}
\hline Opsin & Monkey & $\begin{array}{l}\text { No. of } \\
\text { Injections }\end{array}$ & $\begin{array}{l}\text { Stereotactic coordinate } \\
\text { of first injection } \\
\text { (AP, ML, DV) }\end{array}$ & $\begin{array}{l}\text { Stereotactic coordinate } \\
\text { of last injection } \\
\text { (AP, ML, DV) }\end{array}$ & $\begin{array}{l}\text { No. of units } \\
\text { recorded }\end{array}$ & $\begin{array}{l}\text { No. of significantly } \\
\text { modulated units }\end{array}$ & $\begin{array}{l}\text { No. of } \\
\text { upmodulated } \\
\text { (\% out of sig) }\end{array}$ & $\begin{array}{l}\text { No. of } \\
\text { downmodulated } \\
\text { (\% out of sig) }\end{array}$ & $\begin{array}{l}\text { \% Units recorded } \\
\text { from injection } \\
\text { grid hole }\end{array}$ \\
\hline AAV5-hSyn-ChR2(H134R)-eYFP & B & 8 & $(28.02,15.00,22.54)$ & $(26.08,15.90,19.42)$ & 35 & $29(82 \%)$ & $25(86 \%)$ & $4(14 \%)$ & $100 \%$ \\
\hline AAV5-CAG-ArchT-eGFP & B & 9 & $(27.81,17.43,23.81)$ & $(25.59,17.91,20.24)$ & 33 & $29(87 \%)$ & $0(0 \%)$ & $29(100 \%)$ & $63 \%$ \\
\hline AAV5-CAG-ArchT-eGFP & J & 9 & $(26.67,13.67,24.82)$ & $(25.52,13.73,20.80)$ & 108 & $78(72 \%)$ & $11(15 \%)$ & $67(85 \%)$ & $95 \%$ \\
\hline AAV5-hSyn-eNpHR3.0-eYFP & J & 9 & $(26.07,15.42,24.90)$ & $(25.06,15.72,21.23)$ & 37 & $27(72 \%)$ & $4(15 \%)$ & $23(85 \%)$ & $100 \%$ \\
\hline AAV5-CaMKII-ChR2(E123A)-mCherry & A & 8 & $(23.37,-16.48,26.97)$ & $(23.36,-14.32,24.26)$ & 85 & $58(68 \%)$ & $53(91 \%)$ & $5(9 \%)$ & $100 \%$ \\
\hline
\end{tabular}

$\mathrm{AP}$, Anteroposterior; ML, mediolateral; DV, dorsoventral.

concerns and suggest that ChR2 stimulation contributes to the initiation of movements, but, in most cases, stimulation evokes subthreshold activity that is not sufficiently strong to evoke a motor response.

\section{Materials and Methods}

Experimental procedures. All procedures conformed to local and National Institutes of Health guidelines, including the National Institutes of Health Guide for Care and Use of Laboratory Animals. All experiments were performed with the approval of the California Institute of Technology Institutional Animal Care and Use Committee and the California Institute of Technology Institute Biosafety Committee.

FEF targeting. The FEF was identified by anatomical landmarks in three macaque monkeys (males). Recording chamber placement and electrode trajectories were planned with Planner (Ohayon and Tsao, 2012), a custom-designed software for MRI-guided electrophysiology. Chambers were not aligned to stereotactic coordinates, hence, electrode trajectories span multiple anteroposterior slices (Table 1).

Constructs and viral injection. Five different constructs with comparable titers were used in this study. AAV5-hSyn-eNpHR3.0-eYFP $(3 \times$ $10^{12}$ virus molecules/ml), AAV5-hSyn-ChR2(H134R)-eYFP $\left(4 \times 10^{12}\right.$ virus molecules/ml), AAV5-hSyn-ChR2(E123A)-eYFP (4 × $10^{12}$ virus molecules/ml), and AAV5-CaMKII-ChR2(E123A)-mCherry $\left(4 \times 10^{12}\right.$ virus molecules $/ \mathrm{ml}$ ) were obtained from the Deisseroth Laboratory (Stanford University). AAV5-CAG-ArchT-eGFP $\left(1 \times 10^{12}\right.$ virus molecules $/ \mathrm{ml}$ ) was obtained from the Boyden Laboratory (Massachusetts Institute o Technology). Constructs were packaged at the vector core facility at the University of North Carolina.

We injected $1 \mu \mathrm{l}$ of virus for each $0.5 \mathrm{~mm}$ of cortex. Injections were restricted to sites in which single units were found and saccades could be evoked (with the exception of eNpHR3.0 site in monkey B). Capillary tubing (360 $\mu \mathrm{m}$ outer diameter; TSP200350; Polymicro) was filled with paraffin oil (Omega) using a $100 \mu$ l gas-tight syringe (1710TTL; Hamilton) and a microinjection pump (UMP3 and SYS-MICRO4; WPI). Viral vectors were injected using a 32 gauge injection needle (point style 4; Hamilton) connected to an elbow joint (C360-205; LabSmith), which was connected to a micromanipulator drive (MO-97A; Narishige). A 23 gauge guide tube was used to penetrate the dura and was placed in a grid (Crist Instruments) sitting in the cranial chamber. The needle was slowly lowered $(0.25 \mathrm{~mm} / \mathrm{min})$ to the injection site, and $1 \mu \mathrm{l}$ was injected at a rate of $40 \mathrm{nl} / \mathrm{min}$. We monitored the injected quantity using a blue food dye (Esco Foods) preloaded into the tubing. We then waited $10 \mathrm{~min}$ for the virus to diffuse before slowly lowering the needle and repeating the procedure. All viral injections were performed in the same day in monkeys J and A. Monkey B went through two viral injection sessions separated by 6 months. Injections took place while animals were sedated $(0.02$ $\mathrm{mg} / \mathrm{kg}$ Dexdormitor).

Monkeys J and B were injected with AAV5-hSyn-ChR2(H123R)-eYFP, a membrane channel that excites neurons during blue light illumination (Boyden et al., 2005), AAV5-hSyn-eNpHR3.0-eYFP (Natronomonas pharaonis halorhodopsin), a chloride pump that inhibits neurons during yellow or green light illumination (Gradinaru et al., 2008, 2010), and AAV5CAG-ArchT (Archaerhodopsin), a proton pump that inhibits neurons dur- ing green light illumination (Chow et al., 2010; Han et al., 2011). Monkey A was injected with AAV5-hSyn-ChR2(E123A), a mutant ChR2 with faster channel kinetics, and AAV5-CaMKII-ChR2(E123A), a mutant ChR2 with faster channel kinetics under a cell-type-specific promoter for excitatory neurons (Gunaydin et al., 2010; Mattis et al., 2012).

Monkey A was first tested for neural modulations 2 weeks after injection (modulations found) and was tested for paired stimulation (optical, electrical) 3 weeks after injection (for 2 weeks). Monkey B was first tested for neural modulations 3 weeks after injection (modulations found) and tested for paired stimulation 3.5 weeks after injection (for several months). Monkey J was first tested for neural modulations 1 month after injection (modulations found) and tested for paired stimulation 5 weeks after injection (for several months).

Optical stimulation. Optic fibers (BFL 22-200; Thorlabs) with furcation tubing were stripped for $10-15 \mathrm{~cm}$ using special stripping tools (FTS3, T16S31; ThorLabs) to expose the core. Super glue (modified ethyl cyanoacrylate; Dymax 222/3; Dymax Corporation) was used to attach electrodes at an offset of $0.5-1 \mathrm{~mm}$ relative to the tip of the optic fiber. Light was delivered using laser (diode-pumped solid state, 473 and 532 $\mathrm{nm}$; Shanghai Lasers) coupled to optic fibers using FC/PC connectors (ADAFC2-PMN; Thorlabs). Laser light levels were measured before each experiment using a power meter (PM100D, S130C; Thorlabs).

Electrophysiology. Neural signals were recorded using Plexon (MAP; Plexon). Local field potentials (LFPs) were filtered at $0.7-300 \mathrm{~Hz}$, and single units and multiunits were filtered at $0.15-8 \mathrm{kHz}$ and recorded at 40 $\mathrm{kHz}$. Low impedance electrodes (50-100 k $\Omega$; UEWLEJSMAN1G; FHC) were used for recording and stimulation. Impedance was measured before experiments (NanoZ; White Matter). Units were sorted offline semiautomatically using KlustaKwik (Harris et al., 2000). Units were classified as multiunit if $>2 \%$ of the recorded spikes had an interspike interval smaller than $2 \mathrm{~ms}$. A unit was defined as significantly modulated by light if the average number of spikes during baseline was significantly different (two-tailed paired $t$ test) compared with the average number of spikes during the stimulation period. Baseline period was matched for the duration of optical stimulation to ensure equal variance. Optical stimulation latencies were defined as the first time point in which average firing rate (smoothed using a Gaussian kernel, $\sigma=2 \mathrm{~ms}$ ) exceeded four times the SD during baseline. For the analysis of firing rate changes (see Fig. 2a), three recording sessions were discarded (two in monkey B and one in monkey J) in which multiple optrodes were lowered to two sites simultaneously. The majority of units were collected from the same grid hole used for the viral injection (Table 1).

Electrical microstimulation. Electrical microstimulation was delivered using a current isolator (A365; WPI) and monopolar electrodes with impedance of $50-100 \mathrm{k} \Omega$ (FHC). Biphasic pulses were delivered at 300 $\mathrm{Hz}$, with each pulse lasting $0.15-0.25 \mathrm{~ms}$ with $0.15 \mathrm{~ms}$ interpulse separation, cathodal leading. Currents varied between 10 and $90 \mu \mathrm{A}$. Pulses were generated with custom hardware (Arduino Due), and pulse jitter was $<5 \mu$ s.

Eye movements, saccades, and task. Monkeys were head fixed and passively viewed a screen (Dell P1130; Dell Computers) in a dark room. A small fixation spot $\left(0.25^{\circ}\right.$ in diameter $)$ was presented in the center of the screen. Eye position was monitored during the experiments using an 
iScan system running at $120 \mathrm{~Hz}$. Juice rewards were delivered every 2-3.5 $s$ when the monkey fixated the dot. Juice reward was still delivered if monkeys broke fixation for periods shorter than $300 \mathrm{~ms}$ (allowing blinks or saccades evoked by electrical microstimulation). Eye traces were aligned in time to stimulation onset and in space to the position of the eye at $t=0$ [i.e., $x(t=0)=y(t=0)=0$ ]. We defined the baseline interval of a trial to be $150 \mathrm{~ms}$ before stimulation onset to $50 \mathrm{~ms}$ after stimulation and discarded stimulation trials during which there was no stable baseline, typically corresponding to eye blinks or saccades just before the stimulation trial. For measuring saccade amplitude, eye position was defined as follows: $\sqrt{(x)^{2}+(y)^{2}}$. Circular statistics were computed with CircStat (Berens 2009). Saccade direction and amplitude were estimated by averaging the instantaneous measures at 150-200 ms relative to stimulation onset. Stimulations (both electrical and optical) were considered successful if they evoked a saccade with amplitude $>1.8$ visual degrees and were within $40^{\circ}$ of the average saccade direction (evoked with the highest electrical current). Saccade latencies were defined as the first time point at which eye position exceeded five times the SD during baseline for $10 \mathrm{~ms}$ continuously.

To determine whether a significant increase in saccade probability occurred during combined optical and electrical stimulation, we used the binomial distribution to estimate likelihood of observing $k$ of $N$ saccades, with baseline success probability in each trial obtained from the fraction of successful saccades during electrical stimulation alone. On average, in each experiment, we delivered $22 \pm 10$ electrical stimulations, $21 \pm 9$ combined electrical and optical stimulations, and $132 \pm 45$ optical stimulations alone. The number of coupled stimulations did not differ statistically from the number of electrical stimulations ( $p>0.5$, paired $t$ test).

To generate normalized current plots, we mapped the lowest current level used to 0 and highest current to 1 and linearly interpolated percentages (please note that a normalized current of 0 does not correspond to 0 $\mu \mathrm{A})$. In half of the trials, stimulation was delivered while the fixation spot was visible, and in the other half of the trials stimulation was delivered after the spot was extinguished (Goldberg et al., 1986). For estimating saccade accuracy, the concentration parameter $\kappa$ was estimated from a von Mises distribution: $f(x \mid \mu, \kappa)=\frac{e^{\kappa \cos (\mu-x)}}{2 \pi I(\kappa)}$, where $\mu$ is the mean saccade direction, and $I$ is the modified Bessel function of order 0 .

MRI/fMRI. Structural MRIs were taken in Siemens $3 \mathrm{~T}$ with a single loop coil. Isotropic $0.5 \mathrm{~mm}$ resolution scans were obtained using the following parameters: TR, $2300 \mathrm{~ms}$; TE, $2.94 \mathrm{~ms}$; 256 slices, field of view (FOV), $128 \mathrm{~mm}$; FOV phase, 100\%; slice thickness, $0.5 \mathrm{~mm}$; bandwidth, $190 \mathrm{~Hz} / \mathrm{Px}$; phase encoding, $F \gg H$; $0 \%$ phase oversampling.

fMRI scans were taken in a Siemens $3 \mathrm{~T}$ with AC88 gradient insert using a custom eight-channel coil. Full brain coverage scans were obtained at $1 \mathrm{~mm}$ isotropic resolution with the following parameters: TR, $2000 \mathrm{~ms}$; TE, $17 \mathrm{~ms}$; number of slices, 54; FOV, $96 \mathrm{~mm}$; FOV phase, $100 \%$; slice thickness, $1 \mathrm{~mm}$; bandwidth, $1860 \mathrm{~Hz} / \mathrm{Px}$; phase encoding, $F$ $\gg H$; $0 \%$ phase oversampling; Parallel Acquisition Techniques 2 with generalized autocalibrating partially parallel acquisitions reconstruction. Before scanning, a contrast enhancing agent was injected to the blood ( 8 $\mathrm{mg} / \mathrm{kg}$ Feraheme). During scanning, the monkey was required to fixate a small white $\operatorname{dot}\left(0.25^{\circ}\right)$ on a gray background while electrical or optical stimulation were applied. Juice rewards were given during rest blocks after $3 \mathrm{~s}$ of continuous fixation and randomly (every 2-4 s) during stimulation blocks (because the monkey could not hold fixation as a result of stimulation). Eye position was monitored using a camera and infrared light (iScan) sampled at $120 \mathrm{~Hz}$.

The following stimulation parameters were used during the fMRI scans: (1) electrical, train rate, $1 \mathrm{~Hz}$; train length, $200 \mathrm{~ms}$; pulse frequency, $300 \mathrm{~Hz}$; pulse width, $250 \mu$ s; electrical current, 50-300 $\mu \mathrm{A}$; pulse shape, rectangular biphasic pulses with $100 \mu$ s separation between pulses; (2) optical, train rate, $1 \mathrm{~Hz}$; train length, $900 \mathrm{~ms}$; pulse frequency, 80 $\mathrm{Hz}$; pulse width, $8 \mathrm{~ms}$; irradiance, $82-381 \mathrm{~mW} / \mathrm{mm}^{2}$.

$f M R I$ designs and data analyses. To determine significance levels, we used the general linear model (GLM) to analyze the time courses. We defined four explanatory variables: (1) rest (interstimulation blocks); (2) optical (optical stimulation alone); (3) electrical (electrical stimulation alone); and (4) combined (electrical and optical stimulation). We mod- eled the combined stimulation blocks with an additional explanatory variable to allow nonlinear interactions between the two stimulation types. Blocks were either 40 or $32 \mathrm{~s}$ long. Thirteen to 20 runs were collected per monkey. Analysis was done using available analysis packages (motion correction, AFNI; GLM fitting, FSFast). Visualizations and data postprocessing were done with custom scripts written in MATLAB (Mathworks) and Freesurfer. Raw signal was converted to percentage change, and the sign was flipped (because of the Feraheme). Data were smoothed with a spatial $1 \mathrm{~mm}$ isotropic Gaussian kernel and with a $1 \mathrm{~s}$ Gaussian kernel along the time axis. ROI analysis was performed using an ROI selected based on anatomical landmarks (known optrode tip position).

Histology. Monkey B was perfused with 4\% PFA (4 L) and then with $4 \%$ PFA plus $10 \%$ sucrose $(1 \mathrm{~L})$. The brain was removed and placed in $4 \%$ PFA and $10 \%$ sucrose for $2 \mathrm{~d}$ and then in $4 \%$ PFA and $20 \%$ sucrose for $4 \mathrm{~d}$. Fifty micrometer full-brain sections of frozen tissue were taken with a microtome (model 860; American Optical Company). Immunohistochemistry primary antibodies included the following: DAPI (1:50,000; D9542; Sigma), anti-NeuN (1:5000; MAB377; Millipore), anti-CaMKII (1:50; sc-13082; Santa Cruz Biotechnology), anti-parvalbumin (PV; 1:5000; P3088; Sigma), and anti-GFAP (1:5000; G3893; Sigma). Sections were incubated with primary antibodies and PGT (PBS, 2 g/L gelatin, $0.25 \%$, and Triton X-100) solution overnight (except anti-CaMKII, which was incubated for $72 \mathrm{~h}$ in a cold room). Sections were triple washed with PBS and then incubated with secondary antibodies for $2 \mathrm{~h}$ : anti-rabbit Cy5 (A10523; Invitrogen) and anti-mouse Cy3 (A10521; Invitrogen). Sections were mounted on glass slides (5075-FR; Brain Research Laboratories) using ProLong Gold Antifade Reagent (P36934; Invitrogen). Slides were imaged with Olympus confocal FV1000 using a UPLSAPO $10 \times, 0.40$ numerical aperture lens.

Custom software for visualization, registration, and annotation was developed in MATLAB. Two-dimensional affine transformation was used to register high-magnification $Z$-stack images to a wide-field fluorescence image. The latter was then registered using another $2 \mathrm{D}$ affine transformation to the corresponding photograph of the frozen tissue block. The entire frozen tissue block was then registered using $3 \mathrm{D}$ affine transformation to the MRI scan using Planner (Ohayon and Tsao, 2012). Two brain series spanning the FEF were used for the quantitative histological analysis: (1) NeuN, DAPI, yellow fluorescent protein (YFP), and GFAP; and (2) CaMKII, PV, YFP, and DAPI.

\section{Results}

\section{Localization of FEF and viral injection}

We localized the FEF in three monkeys according to anatomical landmarks in MRI scans. Within the FEF of each monkey, a recording chamber was implanted (not perpendicular to the cortical surface; Fig. 1, $a$ and $b$, MRI reconstructions aligned to chamber coordinates). Electrode trajectories targeted the anterior bank of the arcuate sulcus.

We electrically stimulated each of the sites before injections and tested whether saccades could be evoked with currents lower than $50 \mu \mathrm{A}$. Saccadic eye movements with characteristics similar to previous studies (Bruce et al., 1985) were evoked by electrical stimulation in seven of eight of the injection grid holes (Fig. 1c,d), with the exception of eNpHR3.0 site in monkey B (which was on the rim of the arcuate sulcus). Electrically evoked saccades started $87.9 \pm 35 \mathrm{~ms}$ after stimulation. We observed saccade amplitudes ranging from 2 to 30 visual degrees across the different sites (Fig. 1d). Saccade direction changed along the penetration (Fig. $1 d$, ChR2 injection in monkey B).

Multiple viral injections were made in each monkey along the penetration trajectory (in each grid hole). Viral injection of different constructs were separated by at least $2 \mathrm{~mm}$ from each other. Each injection was separated by $500 \mu \mathrm{m}$ from neighboring injection sites along the same trajectory (Fig. 1b, inset; see Materials and Methods). 
a
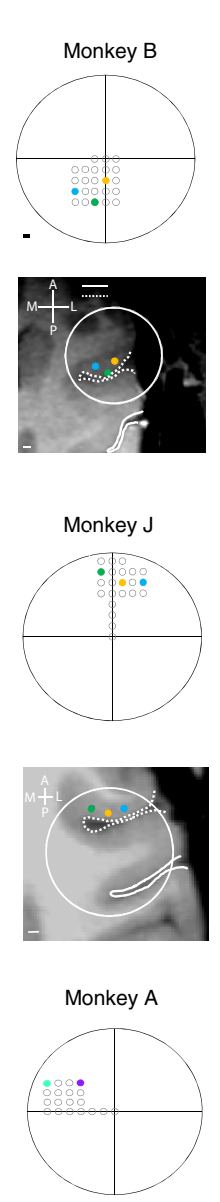

b
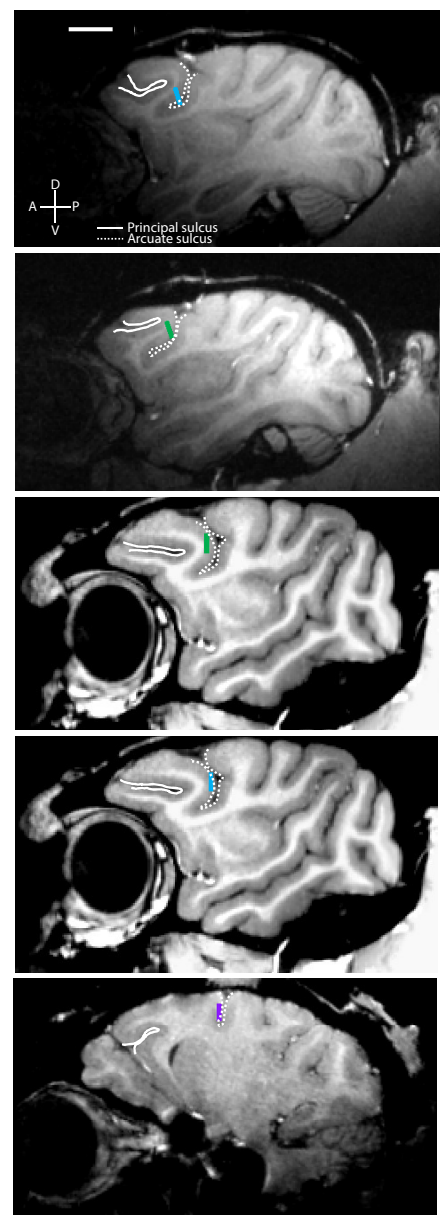
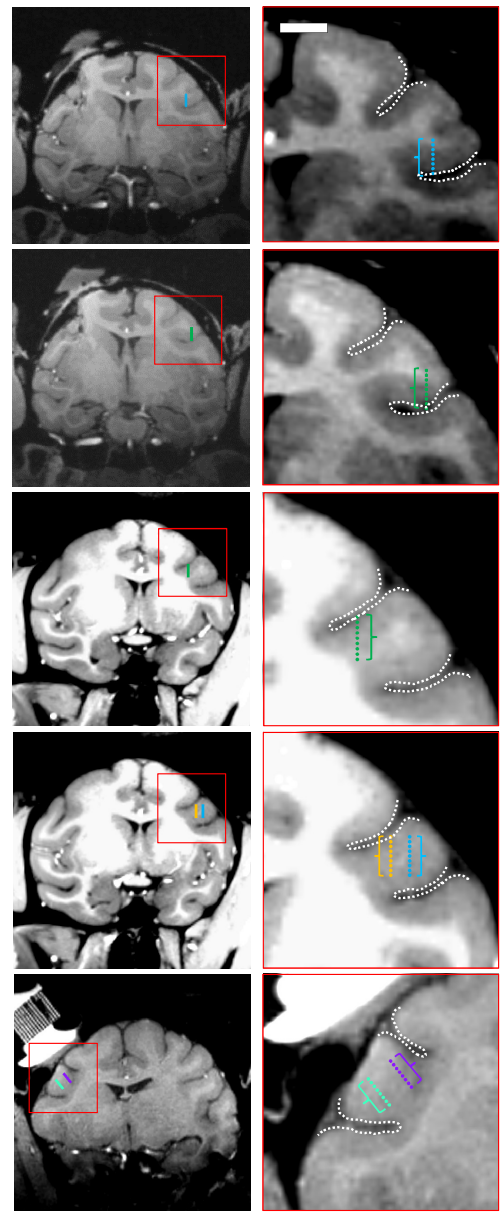

C
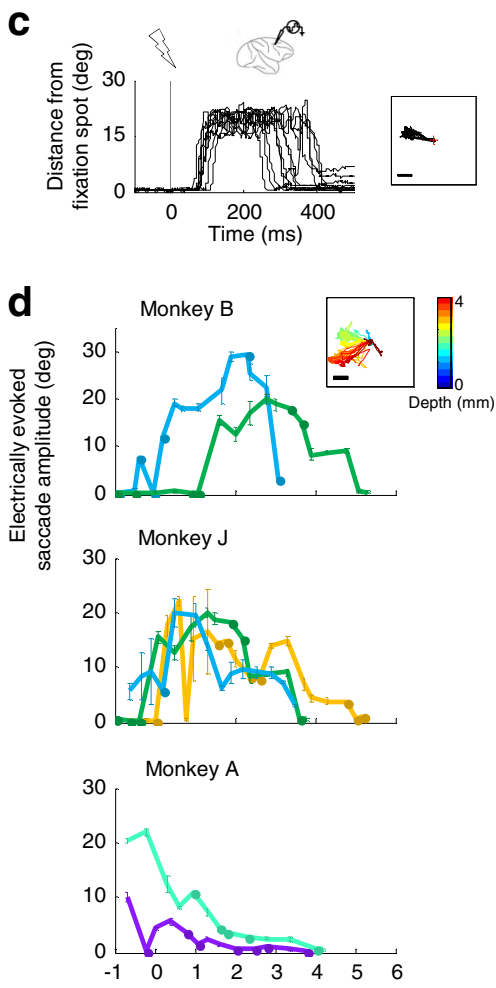

Depth relative to first injection site $(\mathrm{mm})$

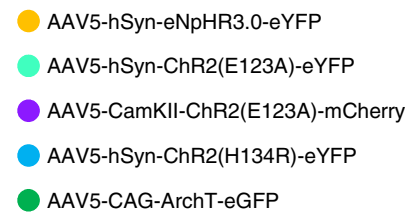

Figure 1. FEF injection sites and electrically evoked saccades. $\boldsymbol{a}$, Top view of the grids used to inject viral vectors and to record neural signals. MRI reconstruction aligned to chamber coordinates is shown below for monkeys B and J. Scale bar, $1 \mathrm{~mm} . \boldsymbol{b}$, MRI reconstructions of injection sites (sagittal and coronal views); eNpHR3.0 injection in monkey B is not shown because of space limitations. Cross-sections are aligned to show the full injection trajectory. Scale bar, $10 \mathrm{~mm}$. Zoom inset, Injection sites (each is represented by a small dot). Scale bar, $5 \mathrm{~mm}$. c, Example of electrically evoked saccades in the ChR2 site in monkey B at a depth of $0.5 \mathrm{~mm}$ relative to the first injection site (distance from fixation spot on the left, eye trace on the right). Eye trace is aligned to electrical microstimulation (delivered at time 0 ). $\boldsymbol{d}$, Average saccade amplitude evoked by electrical microstimulation at various depths in all injection sites (mean \pm SEM). Small inset on the top plot shows all evoked saccades as a function of depth in the ChR2 site in monkey B before viral injection (depth is color coded).

\section{Cells are strongly modulated by light}

Two to 4 weeks after viral injections, we began recordings to characterize light-induced neural activity using custom-built optrodes (electrode glued to an optic fiber). We characterized hSyn-ChR2(H134R) sites (monkeys J and B) with $473 \mathrm{~nm}$ laser and tested neural responses to continuous light $(500-2000 \mathrm{~ms}$, irradiance $<318 \mathrm{~mW} / \mathrm{mm}^{2}$ ) or short pulses $(5-8 \mathrm{~ms}, 40-80 \mathrm{~Hz}$, irradiance $<318 \mathrm{~mW} / \mathrm{mm}^{2}$ ). Figure $2 a$ depicts the response of an example single unit from monkey B to a $500 \mathrm{~ms}$ pulse of blue light. The unit significantly increased firing rate compared with baseline activity measured $1 \mathrm{~s}$ before stimulation $(p<0.001$, two-tailed paired $t$ test). Spike wave form (Fig. $2 a$, top right) remained the same, indicating that the response was not attributable to other cells or to an electrically induced artifact. The observed reduction of firing rate during the stimulation interval was attributed to our laser instability and was correlated with the output, as measured with a power meter (Fig. $2 a$, bottom right inset).

Similar neural modulations were observed in both monkeys, and statistics were pooled for plotting purposes (statistics for each monkey in each injection site are given in Table 1). Of 184 recorded single units, $78 \%$ of the units significantly modulated their response (Fig. $2 b$, leftmost column). The majority of units increased their responses (117 of 144 modulated units), and the population was significantly upmodulated $(p<0.001$, two-tailed paired $t$ test). Of the 144 modulated units, 126 were probed with a $500 \mathrm{~ms}$ continuous pulse of light and showed an increase in firing rate. The average response of those units followed the same response as the unit shown in Figure $2 a$ (see also Fig. $2 c$, top). Similar to previous studies (Han et al., 2009; Diester et al., 2011), we observed a light-induced artifact in the LFP. A strong downward deflection to light onset and strong upward deflection when the light was turned off were observed (Fig. $2 c$, bottom), likely attributable to the Becquerel effect (Han, 2012). The mean response latency was $2.84 \mathrm{~ms}$ (Fig. $2 d$, latencies shown only up to 20 $\mathrm{ms}$ ), with the majority of units responding within $1 \mathrm{~ms}$. We found that 27 recorded units showed a reduction in firing rate, which could be attributable to secondary network effects or to spread from a neighboring viral injection of an inhibitory opsin.

Qualitatively similar responses were observed in hSynChR2(E123A) and CaMKII-ChR2(E123A) sites (Fig. 2, second and third columns); $74 \%$ of recorded units modulated their response, and responses were similar in shape, amplitude, and latencies (Fig. 2, second and third columns; Table 1). The 

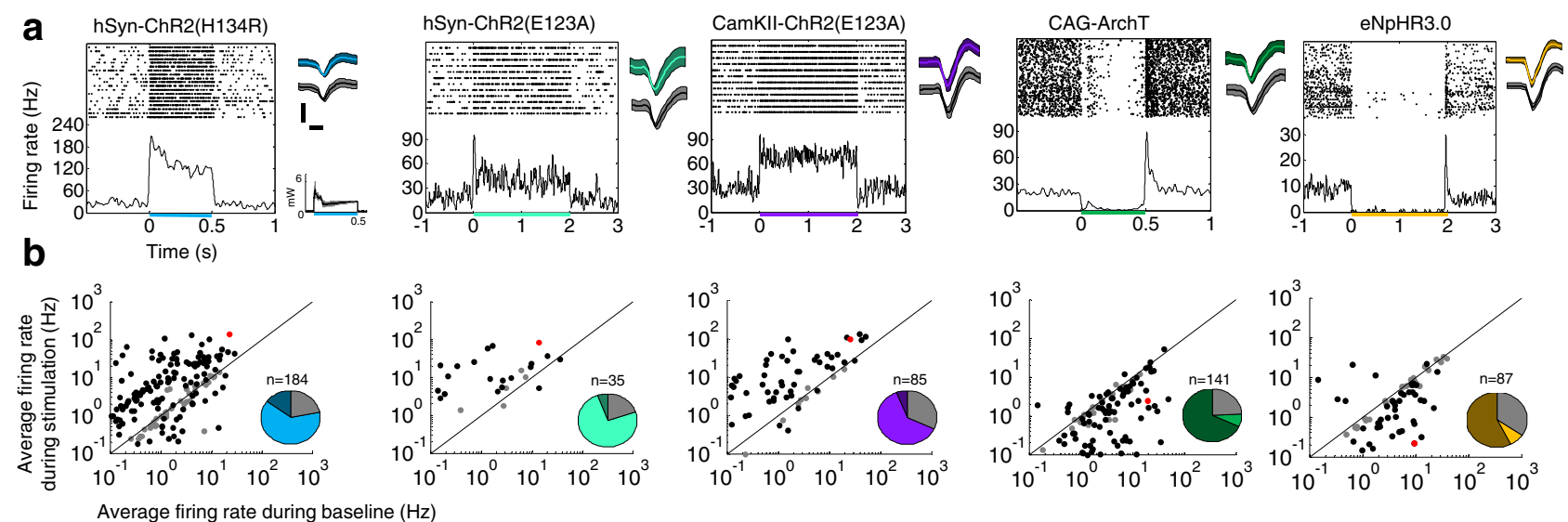

Average firing rate during baseline $(\mathrm{Hz}$

C
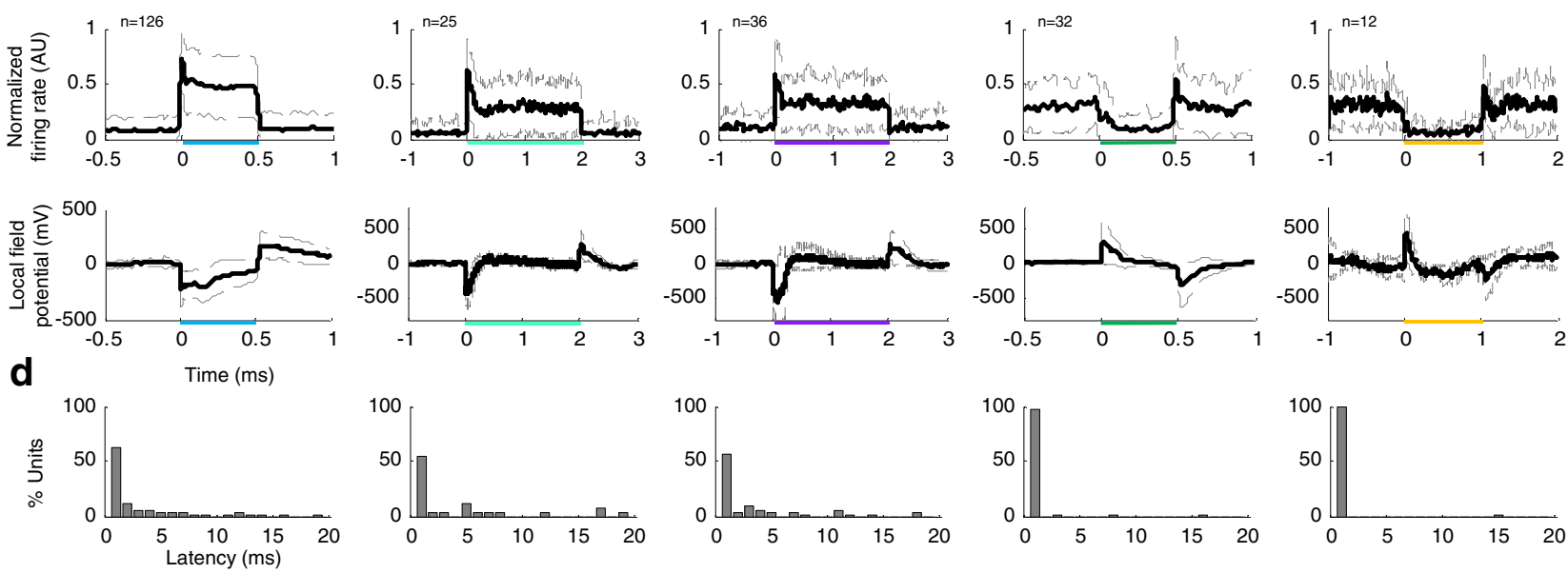

Figure 2. Electrophysiological characterization of light-induced modulation of neural activity in the FEF. $\boldsymbol{a}$, Peristimulus time histogram for a single unit recorded from each injection site and the corresponding raster plot (shown above). Stimulation interval is denoted by the colored bar. Traces on the right of each plot show the average spike wave (mean \pm SD) form during stimulation (in color) and before and after stimulation (in gray). Calibration: $250 \mu \mathrm{V}, 250 \mu \mathrm{s}$. Bottom right small inset, Measured laser output intensity. $\boldsymbol{b}$, Scatter plot of average firing rate during the stimulated interval compared with baseline activity across all recorded cells in all injection sites (data pooled across monkeys). Monkeys were required to maintain fixation on a small dot. Juice rewards were delivered every $2-4 \mathrm{~s}$ if the monkey did not break fixation. Black dots denote significant modulation compared with baseline ( $p<0.05$, two-tailed paired $t$ test), gray dots denote nonsignificant modulation, and example cell shown in $\boldsymbol{a}$ is highlighted in red. Pie charts denote the fraction of non-modulated units (gray), upmodulated units (bright color), and downmodulated units (dark color). c, Average unit responses to continuous light (mean \pm SD; top, normalized firing rate; bottom, LFP). Average is over modulated units only (upmodulated for ChR2 and downmodulated for ArchT and eNpHR3.0). $\boldsymbol{d}$, Histogram of response latencies.

ChR2(E123A) mutant was shown to evoke higher firing rates in vivo in rodents (Mattis et al., 2012), and indeed, we found a small but significant increase in the cumulative distribution of firing rate ratio $(p=0.03$, two-sample, one-tailed KolmogorovSmirnov test).

We illuminated CAG-ArchT and hSyn-eNpHR3.0 sites with $532 \mathrm{~nm}$ laser by delivering continuous light (500-2000 ms). Firing rate was almost completely eliminated during the stimulation period and quickly recovered when the light was turned off (Fig. $2 a$, single-unit examples, fourth and fifth columns). The majority of units were strongly silenced $(68 \%$ of all recorded units in ArchT sites and 52\% in eNpHR3.0 sites; Table 1, Fig. $2 b$, fourth and fifth columns). In both ArchT and eNpHR3.0 sites, we observed a positive deflection of the LFP to light onset (Fig. $2 c$, fourth and fifth columns) and a negative deflection when the light was turned off.

Optical stimulation coupled to low current electrical stimulation increases probability of evoked saccades In the work of Han et al. (2009) and Gerits et al. (2012), optical stimulation in FEFs expressing ChR2 did not evoke saccades, although strong firing rate changes were observed. Thus, it re- mained unclear whether such perturbations can contribute to the generation of a saccadic eye movement.

To test whether FEF optical stimulation contributes to generation of saccadic eye movements, we probed FEFs with both optical and electrical stimulation. Although high current electrical stimulation reliably evokes saccades (Fig. $1 c, d$ ), low current electrical stimulation reduces the probability of evoking a saccade without significant changes to saccade amplitude or velocity (Bruce et al., 1985). Thus, one direct way to test whether optical stimulation contributes to generation of saccadic eye movement is to deliver at the same time low current electrical stimulation and optical stimulation and evaluate whether the probability of evoking saccades changes compared with low current electrical stimulation alone.

While monkeys passively fixated a small dot, we lowered an optrode until eye movements could be evoked with low current electrical stimulation $(<50 \mu \mathrm{A})$, and single units were modulated with light. A stimulation pulse (optical or electrical) was considered successful in evoking a saccade if eye position changed $>1.8^{\circ}$ ( similar results were obtained using a higher threshold of $5^{\circ}$; see Materials and Methods). For example, a $25 \mu \mathrm{A}$ electrical stimulation in the ChR2 site in monkey $\mathrm{B}$ (at $1 \mathrm{~mm}$ depth relative to the 
a

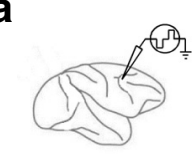<smiles>CC1(C)C2CC3CC(C3)C1C2</smiles><smiles>CC(C)(C)C12CC3CC(CC(C3)C1)C2</smiles>
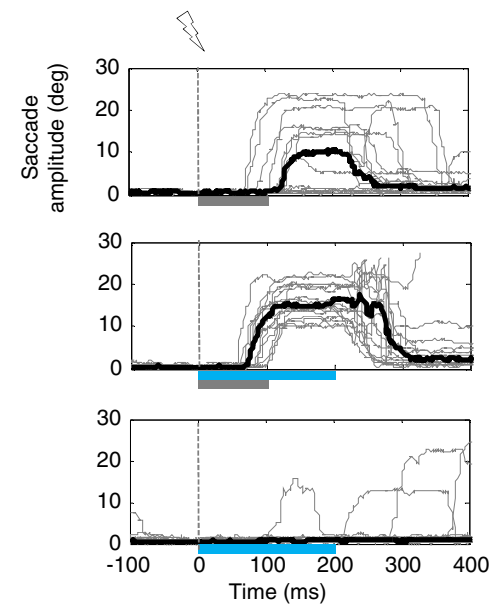

b
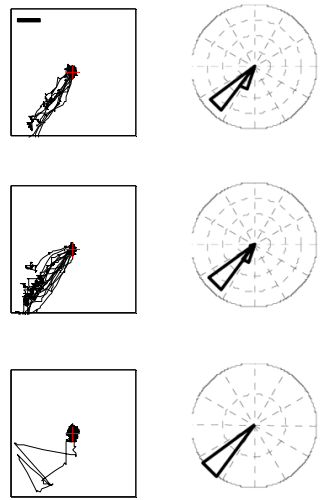

C
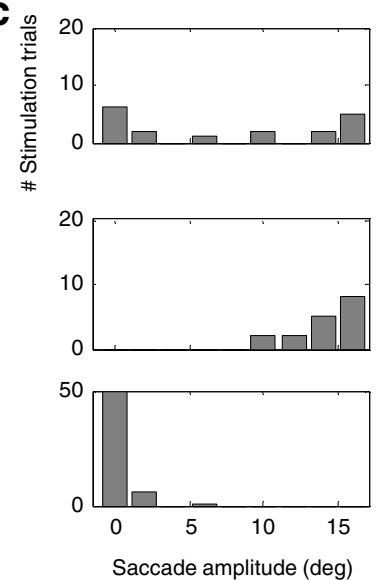

g

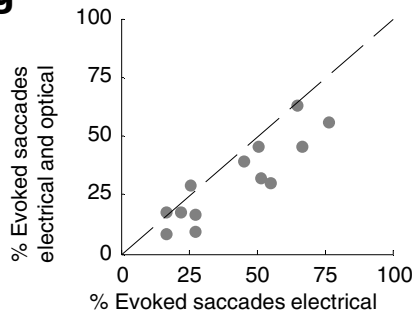

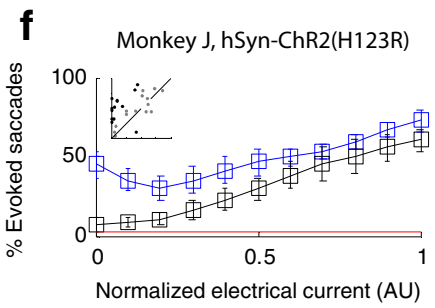
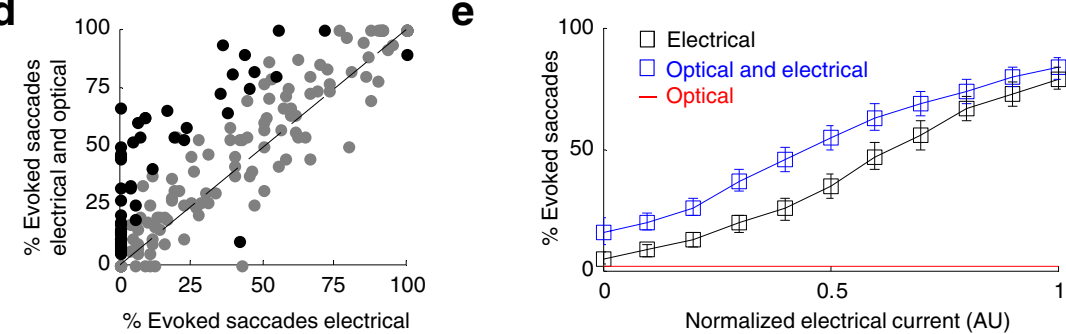

Monkey B, hSyn-ChR2(H123R)
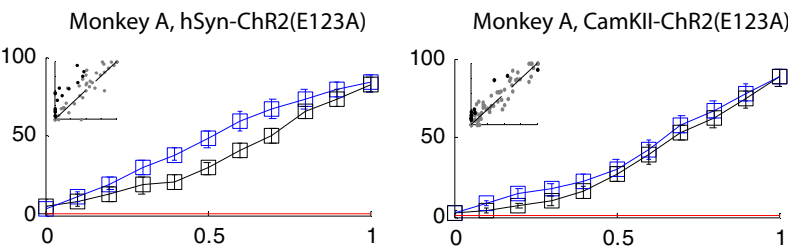

Figure 3. Optical and electrical stimulation in ChR2 sites. $a$, Schematics of three different stimulation configurations: electrical (top), electrical and optical (middle), optical (bottom). The right panels show eye position traces from an example ChR2 site in monkey B elicited by the three stimulation configurations (relative to onset of stimulation at $t=0$ ). Solid black curve represents the median trace of all trials, including stimulation trials that did not evoke saccades (note that it does not represent the average amplitude of evoked saccades). Configuration 1, Electrical stimulation, denoted as a gray bar below the plot ( $100 \mathrm{~ms}, 300 \mathrm{~Hz}, 0.25 \mu$ s biphasic, $25 \mu \mathrm{A}$ ); Configuration 2, coupled optical stimulation (200 ms continuous pulse, $190 \mathrm{~mW} / \mathrm{mm}^{2}$, denoted as blue bar below plot) and electrical stimulation (same parameters as Configuration 1); Configuration 3, optical stimulation (200 ms continuous pulse, $190 \mathrm{~mW} / \mathrm{mm}^{2}$ ). $\boldsymbol{b}$, Left, Eye position for all stimulation trials. Scale bar, $5^{\circ}$. Right, Normalized polar histogram for trials with evoked saccades. c, Histogram of average saccade amplitude after stimulation (measured at $150-200$ ms relative to stimulation onset). $\boldsymbol{d}$, Scatter plot of the percentage of evoked saccades for combined optical and electrical stimulation versus electrical stimulation alone. Each dot represents a single experiment (multiple stimulations) performed with a fixed electrical current and fixed irradiance. Data are shown for all four ChR2 sites in three monkeys. Black dots indicate experiments with a significant difference ( $p<0.01$, binomial test; see Notes), and gray dots indicate not significant. $e$, Percentage of successfully evoked saccades by electrical stimulation (black curve), combined optical and electrical stimulation (blue curve), and optical stimulation alone (red curve). Data are pooled across three monkeys and three injection sites (hSyn promoter). Electrical currents were normalized by the minimal and maximal currents used in each site (see Notes). Error bars denote SEM. $\boldsymbol{f}$, Similar to $\boldsymbol{d}$ and $\boldsymbol{e}$, but data are plotted for each injection site separately. $\boldsymbol{g}$, Similar to $\boldsymbol{d}$ but for control sessions in which the optic fiber was outside the brain.

first injection) evoked saccades in only 10 of 18 stimulation attempts (Fig. $3 a-c$, top row). When the same electrical current was coupled to optical stimulation, a significant increase in the number of saccades was observed $(p=0.0003$, binomial test; Fig. $3 a-c$, middle row): $100 \%$ (17 of 17 ) of stimulations were successful in evoking a saccade. Only one saccade was observed following optical stimulation without electrical stimulation, which could be attributed to random eye movements of the monkey (Fig. $3 c$, bottom row).

We define an experiment as a set of consecutive trials during which stimulation type (optical, electrical, or both) was randomly interleaved while stimulation parameters (current and irradiance) were held fixed. In each experiment, we delivered on average $22 \pm 10$ electrical stimulations, $21 \pm 9$ combined electrical and optical stimulations, and $132 \pm 45$ optical stimulations alone.

We repeated experiments at various depths along the penetration and considered each a separate site (Table 2). Within each site, we varied the level of electrical current, ranging from values that did not evoke any saccades $(\sim 10 \mu \mathrm{A})$ to values that reliably evoked saccades $(\sim 50-90 \mu \mathrm{A})$. The scatter plot in Figure $3 d$ shows the percentage of evoked saccades in all recorded experiments (regardless of electrical current used) in all three monkeys. We found that 50 of 193 experiments resulted in a significant change in the probability of evoking saccades when optical stimulation was coupled to electrical stimulation $(p<0.01$, binomial test; see Materials and Methods). A significant increase was found in all three monkeys (Table 2), and the average percentage of evoked saccades with optical and electrical stimulation was significantly higher than with electrical stimulation alone $(p<$ $0.001, n=193$, paired right-tailed $t$ test).

In accord with previous studies, we found a monotonic increase in the probability of evoked saccades as the electrical current amplitude increased (Fig. $3 e$, data pooled across all hSyn sites in three monkey; Fig. $3 f$, data plotted for each viral injection 
Table 2. List of the number of stimulation experiments, latencies of evoked saccades, and $p$ value indicating whether the latency distribution during combined stimulation significantly differed compared with latencies of saccades evoked with electrical stimulation alone (Kolmogorov-Smirnov test)

\begin{tabular}{|c|c|c|c|c|c|c|c|}
\hline Opsin & Monkey & $\begin{array}{l}\text { No. of sites probed } \\
\text { with electrical and } \\
\text { optical stimulation }\end{array}$ & $\begin{array}{l}\text { No. of stimulation } \\
\text { experiments (at } \\
\text { various currents) }\end{array}$ & $\begin{array}{l}\text { No. of experiments } \\
\text { showing significant } \\
\text { modulation }\end{array}$ & $\begin{array}{l}\text { Latency } \\
\text { (electrical) }\end{array}$ & Latency (electrical and optical) & Latency $p$ value \\
\hline AAV5-hSyn-ChR2(H134R)-eYFP & B & 3 & 20 & $7(35 \%)$ & $98.36 \pm 24.60$ & $91.13 \pm 23.67$ & 0.0017 \\
\hline AAV5-hSyn-ChR2(H134R)-eYFP & J & 5 & 27 & $13(48 \%)$ & $115.38 \pm 29.52$ & $106.88 \pm 24.26$ & 0.0038 \\
\hline AAV5-hSyn-ChR2(E123A)-eYFP & $A$ & 9 & 58 & $16(28 \%)$ & $106.84 \pm 31.72$ & $104.11 \pm 20.77$ & 0.22935 \\
\hline AAV5-CaMKII-ChR2(E123A)-mCherry & $A$ & 13 & 88 & $14(16 \%)$ & $100.32 \pm 21.26$ & $100.90 \pm 21.10$ & 0.8334 \\
\hline AAV5-CAG-ArchT-eGFP & B & 5 & 35 & $5(14 \%)$ & $103.14 \pm 26.24$ & $103.50 \pm 27.35$ & 0.48456 \\
\hline AAV5-CAG-ArchT-eGFP & $\mathrm{J}$ & 5 & 37 & $7(19 \%)$ & $97.50 \pm 25.42$ & $95.71 \pm 22.14$ & 0.29592 \\
\hline
\end{tabular}
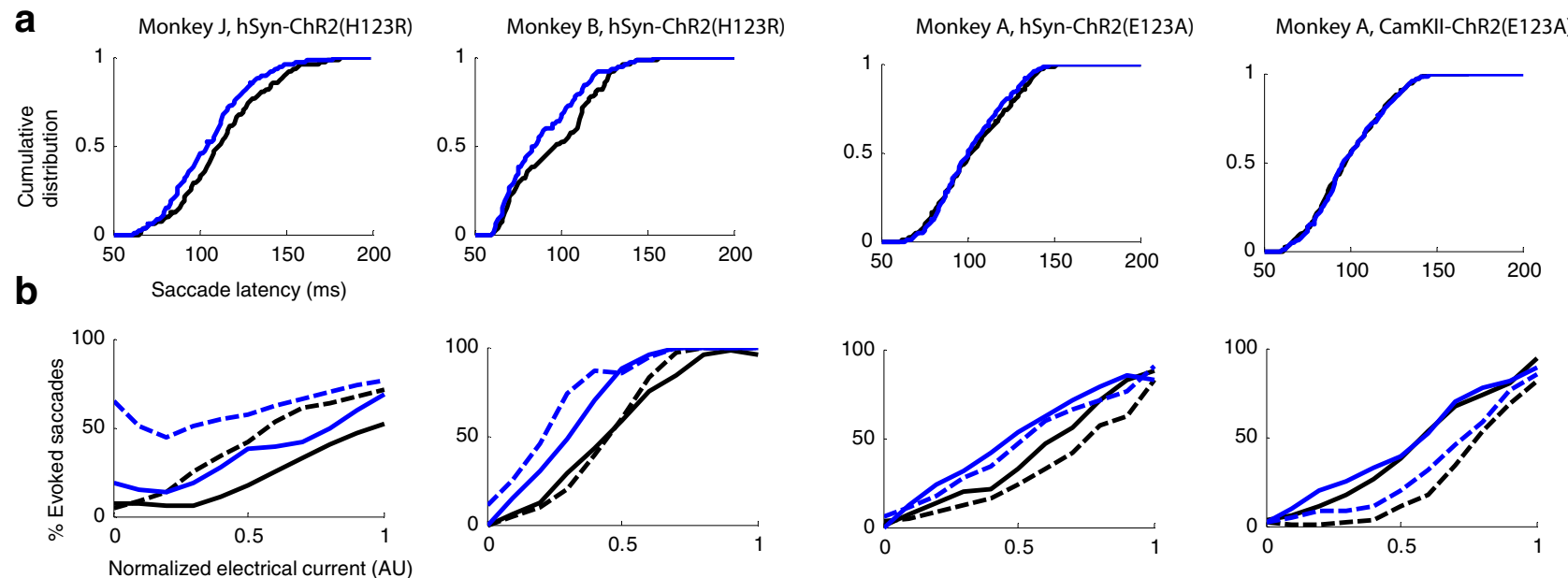

C

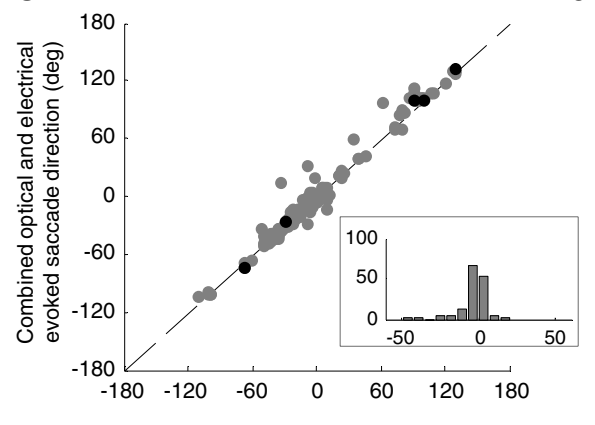

Electrical evoked saccade direction (deg)

d

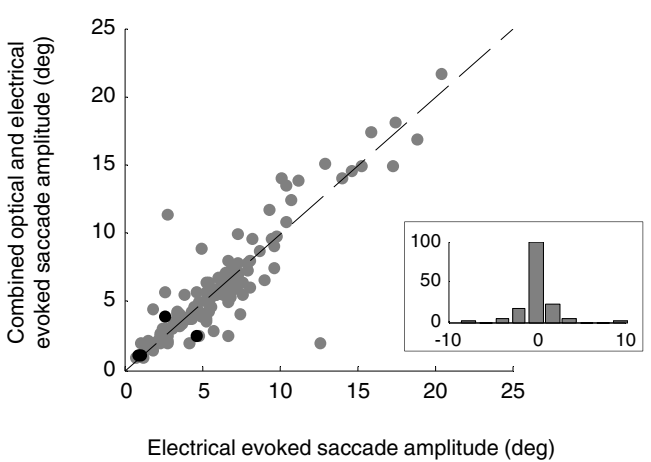

Figure 4. Optical modulation of saccade parameters. $\boldsymbol{a}$, Cumulative distribution of saccade latencies (data shown for each ChR2 injection site). Blue curve, Combined electrical and optical stimulation; black curve, electrical stimulation. $\boldsymbol{b}$, Effectiveness of stimulation when the fixation dot was on the screen (solid curves) or when it was extinguished just before stimulation (dotted curves). $\boldsymbol{c}$, Scatter plot of mean saccade direction for combined optical and electrical stimulation versus electrical stimulation alone. Black dots indicate experiments with a significant difference in direction ( $p<0.01$, nonparametric test for equal medians), and gray dots indicate not significant. Small inset shows the difference in saccade direction across all recorded experiments. $\boldsymbol{d}$, Scatter plot of mean saccade amplitude. Conventions same as in c. Black dots indicate a significant change in amplitude ( $p<0.01$, Mann-Whitney test).

separately). One may expect that the effect of optical stimulation will be more pronounced during experiments in which the electrical stimulation was low, and indeed we found that the increase in saccade probability during combined stimulation was larger for low electrical currents (Fig. 3e,f). Furthermore, when the electrical current was set to the lowest level for which no saccades were evoked (but still higher than $0 \mu \mathrm{A}$ ), the addition of optical stimulation still increased the probability and saccades were observed during combined stimulation (15 significant experiments; points along the $y$-axis in Fig. $3 d$ ). However, in all these experiments, optical stimulation alone did not evoke any significant number of saccades $(0.96 \pm 1.55 \%$, one-sided binomial test) compared with the number of saccades that would be expected by chance (attributable to random gaze shifts).
One concern with measuring behavior when using optical stimulation is that light leakage from the optic fiber might influence the monkey's behavior. Although we sealed the chamber and surrounds to prevent light leakage, the possibility remained that the observed eye movements were attributable to the monkey noticing light from the optic fiber and redirecting his gaze upward. To control for this, we ran several experiments in which an electrode was lowered to the FEF and an optic fiber was lowered into the chamber but not the brain. We did not find any significant increase in saccade probability during these experiments (Fig. $3 g$ ). Furthermore, the saccades evoked during combined stimulation typically followed the direction evoked by electrical stimulation alone (and not upward, as would be expected from the location of the light source). 
a
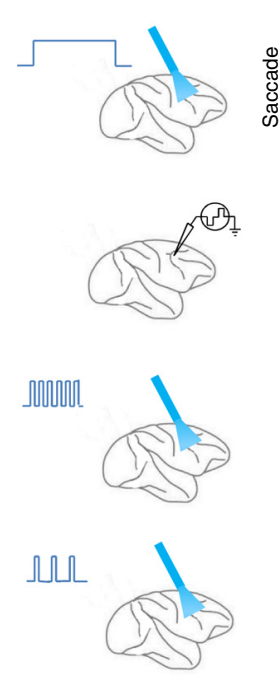

d

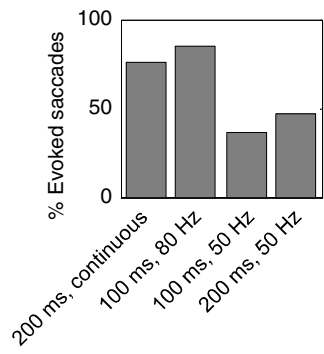

b
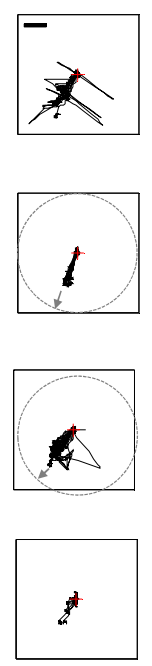

$\begin{array}{rrr}100 & 200 & 300 \\ \text { Time (ms) }\end{array}$

e

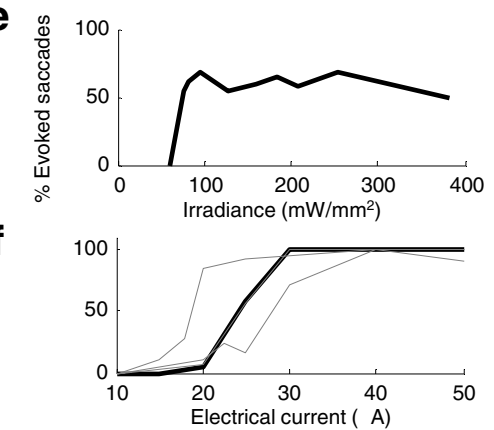

C
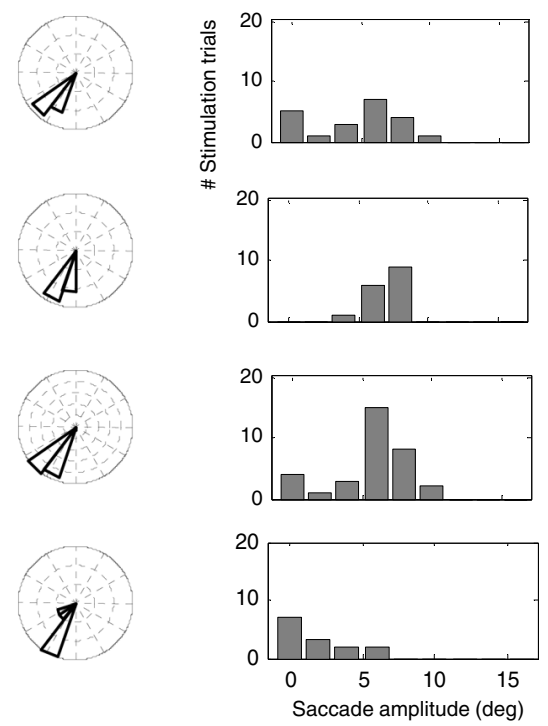

g

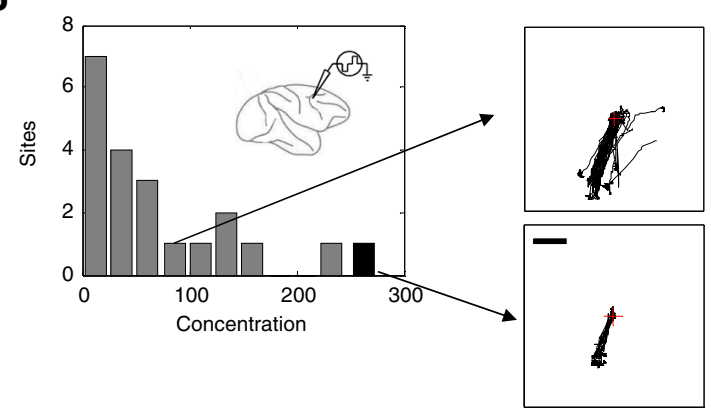

Figure 5. Saccades evoked with ChR2 optical stimulation. $\boldsymbol{a}-\boldsymbol{c}$, Conventions are the same as in Figure 3. First row, Optical stimulation with a $200 \mathrm{~ms}$ continuous pulse. Second row, Electrical stimulation ( $100 \mathrm{~ms}, 300 \mathrm{~Hz}, 0.25 \mu$ s biphasic, $50 \mu \mathrm{A}$ ). Third row, Optical stimulation ( $100 \mathrm{~ms}, 80 \mathrm{~Hz}, 8 \mathrm{~ms}$ pulse). Fourth row, Optical stimulation ( $100 \mathrm{~ms}, 50 \mathrm{~Hz}, 8 \mathrm{~ms} \mathrm{pulse})$. Scale bar, $5^{\circ}$. d, Percentage evoked saccades for four different optical stimulation trains. $\boldsymbol{e}$, Percentage evoked saccades as a function of optical stimulation laser intensity. $\boldsymbol{f}$, Percentage evoked saccades as a function of electrical current. The special site in which saccades were evoked with optical stimulation is shown in black. Three other sites from monkey B are shown in gray. $\boldsymbol{g}$, Histogram of von Mises concentration parameter of electrically evoked saccades across all recorded sessions (gray bars) and the concentration parameter (for electrically evoked saccades) found at the special site from in which optical stimulation alone evoked saccade (black bar). Higher concentration values correspond to smaller saccade angular variance (more accurate saccades). Top right inset shows eye movements with a low concentration parameter $(\kappa=76)$; bottom right inset shows eye movements for the highest concentration, found at the site in which optical stimulation evoked saccades $(\kappa=263)$. Scale bar, $5^{\circ}$.

In two monkeys, saccades evoked by the combined stimulation started significantly earlier compared with saccades evoked with only electrical stimulation (Fig. $4 a, p<0.01$, KolmogorovSmirnov test; Table 2). We also found that stimulation effectiveness in evoking saccades depended on whether the fixation dot appeared on the screen or was extinguished just before stimulation (Fig. $4 b$; see Materials and Methods). In both monkeys J and $\mathrm{B}$, we observed an increase in the efficacy of stimulation (either electrical or combined) when the fixation dot was not displayed (and the opposite case in monkey A).

Of 193 stimulation experiments, we only found five that led to a significant change in saccade direction during combined optical and electrical stimulation compared with the direction observed during electrical alone evoked saccades (Fig. $4 c, p<0.01$, nonparametric multisample test for equal medians using CircStat). The average saccade deviation on those five experiments was $5.75 \pm 2.89^{\circ}$. Similarly, we only observed three experiments in which there was a significant change in saccade amplitude (Fig. $4 d, p<0.01$, Mann-Whitney test), with a small amplitude difference $\left(0.21 \pm 1.47^{\circ}\right)$.

\section{Saccades evoked with optical stimulation alone}

The results so far suggest that optical stimulation contributes to the generation of saccadic eye movement but is not strong enough to initiate a movement without the coupled electrical stimulation. However, we found for one specific site in monkey B (stretching between 2 and $2.5 \mathrm{~mm}$ below the first injection site) that optical stimulation alone could consistently evoke saccades without electrical stimulation (Fig. $5 a-c$, first row). In this example, the success rate of evoking a saccade with a $200 \mathrm{~ms}$ continuous light pulse was $76 \%$ ( $n=43$ stimulation pulses). Saccades had similar onset latencies and amplitudes to those evoked by electrical stimulation at the same site (Fig. $5 a-c$, second row).

We tested various stimulation trains and found that saccades could still be evoked with a shorter train $(100 \mathrm{~ms}, 80 \mathrm{~Hz}, 8 \mathrm{~ms}$ pulse; Fig. $5 a-c$, third row). However, the efficiency of the stimulation decreased with lower pulse frequencies (Fig. $5 a-c$, fourth row, $d$ ), suggesting that high-frequency optical stimulation is a necessary condition for evoking a saccade. However, no optical stimulationevoked saccades were observed in a third monkey injected with second-generation $\mathrm{ChR} 2$, which has improved channel kinetics and 


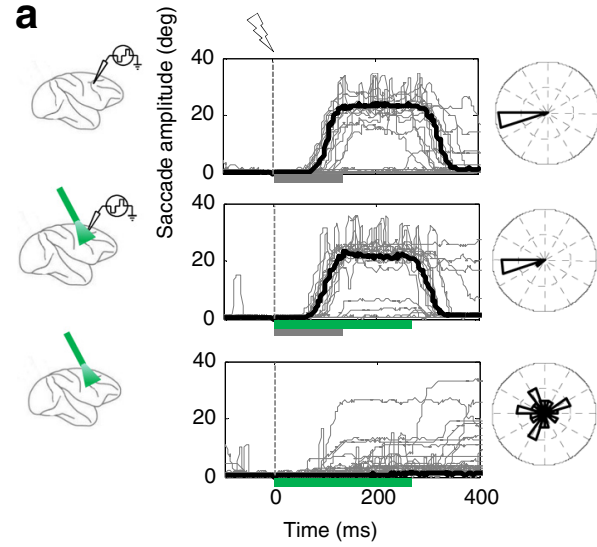

C

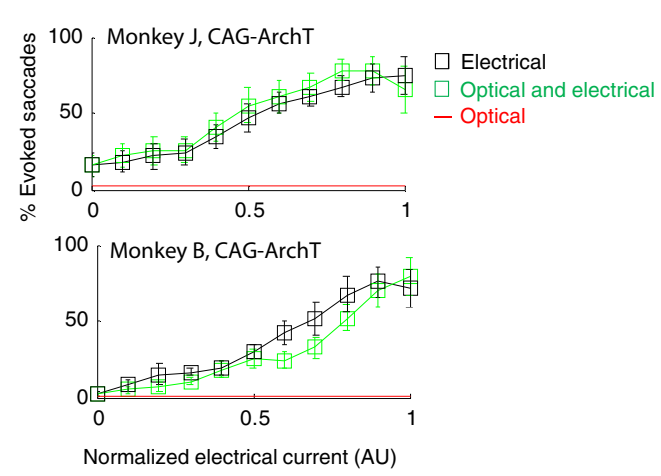

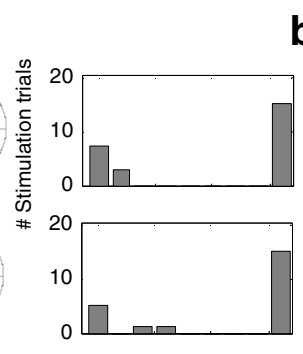

b ฮ
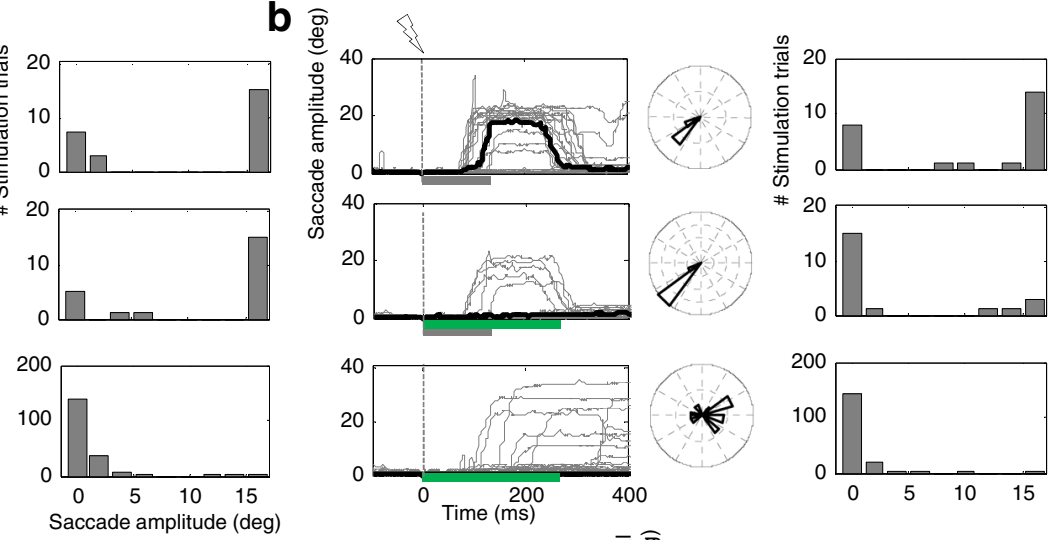
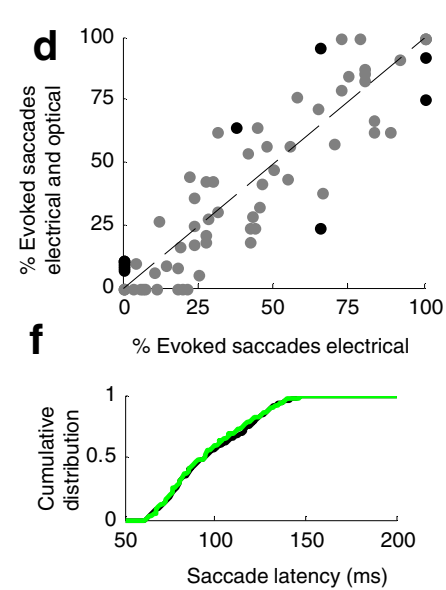
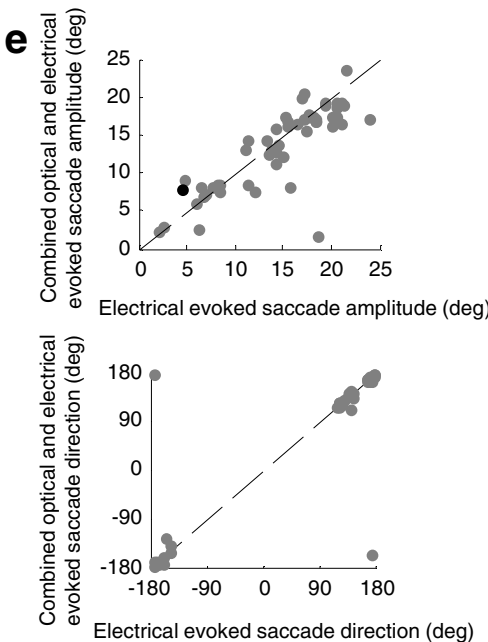

Figure 6. Optical and electrical stimulation in ArchT sites. Conventions same as Figure 3.a, Representative stimulation experiment in monkey J. Top row, Electrical stimulation (100 ms, $300 \mathrm{~Hz}$, $0.25 \mu$ s biphasic, $70 \mu \mathrm{A}$ ); middle row, combined stimulation ( $100 \mathrm{~ms}, 300 \mathrm{~Hz}, 0.25 \mu$ s biphasic, $70 \mu$ A plus $200 \mathrm{~ms}$ continuous optical pulse, $190 \mathrm{~mW} / \mathrm{mm}^{2}$ ); bottom row, optical stimulation ( 200 ms continuous optical pulse, $\left.190 \mathrm{~mW} / \mathrm{mm}^{2}\right)$. $\boldsymbol{b}$, Representative stimulation experiment in monkey B. Electrical stimulation $(100 \mathrm{~ms}, 300 \mathrm{~Hz}, 0.25 \mu$ s biphasic, $25 \mu \mathrm{A})$ and optical stimulation (200 ms continuous optical pulse, $190 \mathrm{~mW} / \mathrm{mm}^{2}$ ). c, Percentage of successfully evoked saccades by electrical stimulation (black curve), combined optical and electrical stimulation (green curve), and optical stimulation alone (red curve). Data shown for each monkey separately. $\boldsymbol{d}$, Scatter plot of the percentage of evoked saccades for combined optical and electrical stimulation versus electrical stimulation alone. Data are pooled from both monkeys. $\boldsymbol{e}$, Average saccade amplitude and direction during optical and combined stimulation. $\boldsymbol{f}$, Cumulative distribution of saccade latencies for electrical stimulation (black trace) and combined electrical and optical stimulation (green).

can be used to drive cells to higher firing rates (Fig. 2). However, stimulating at those sites did evoke a similar increase in saccade probability when coupled to electrical microstimulation (Fig. $3 f$ ).

On a consecutive day, we returned to the special site in monkey $B$ and tested the effect of manipulating laser intensities on the percentage of evoked saccades. We found that increasing the laser intensity led to a quick saturation in the success rate of evoking saccades (60\%; Fig. 5e) and that saccades could be evoked with as little as $82 \mathrm{~mW} / \mathrm{mm}^{2}$.

We also noticed that the direction of saccades evoked by optical stimulation significantly differed from that evoked by electrical stimulation by $16^{\circ}$ (Fig. $5 b$, second and third rows; $p<0.001$, nonparametric multisample test for equal medians using CircStat). This difference may have been caused by the $0.5 \mathrm{~mm}$ offset between the electrode tip and the tip of the optic fiber and indicates that two subpopulations encoding different directions were stimulated.

Optical stimulation was successful in evoking saccades when delivered at a depth of $2-2.5 \mathrm{~mm}$ relative to the first injection but not at other depths (although they could be still evoked with electrical stimulation) or in neighboring grid hole penetrations. There are several possible explanations for this: (1) the effective site contained an especially high concentration of ChR2expressing cells (see histological analysis below); (2) the effective site had some unique physiological properties, for example, lower spike thresholds; and (3) the effective site had some unique functional specificity, for example, a higher concentration of cells encoding the same saccade direction.

We reasoned that, if the site contained neurons with lower spike threshold, then lower electrical currents should still evoke a saccade (compared with currents used in other sites). However, when we probed this site with varying electrical currents, we observed a similar percentage of evoked saccades compared with other sites (Fig. $5 f$ ), suggesting that similar electrical thresholds were needed to evoke a behavior.

We found that electrically evoked saccades in the special site were significantly more accurate in their direction compared with all other sites ( $p<0.01$, one-sided $t$ test; Fig. $5 g$, data pooled across all hSyn sites from three monkeys; see Materials and Methods). Thus, a parsimonious explanation why saccades could be driven optically only at this site is that there was some functional specificity, such as a larger concentration of cells encoding the same saccade direction.

\section{Inhibition of FEFs using ArchT}

We repeated the optical and electrical experiments in ArchT sites to test the effects of optical inhibition on initiation of 

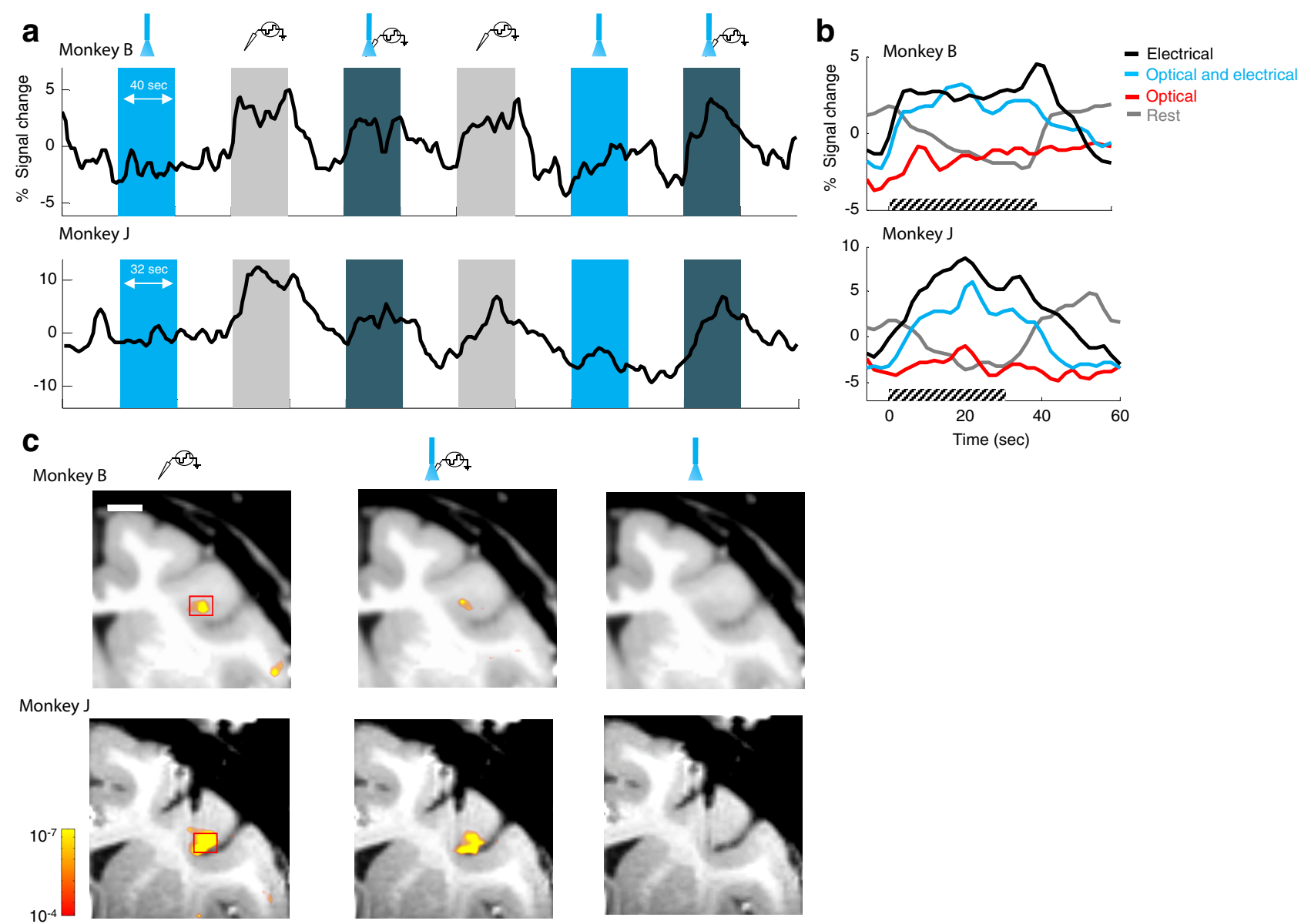

Figure 7. fMRI activity close to the optrode tip during electrical and optical stimulation. $a$, Average fMRI response measured during a block design experiment with conditions: rest (white background), optical stimulation (bright blue background, $1 \mathrm{~Hz}$ train for $900 \mathrm{~ms}$; pulses: $80 \mathrm{~Hz}, 8 \mathrm{~ms}, 82 \mathrm{~mW} / \mathrm{mm}^{2}$ ), electrical stimulation (gray background, $1 \mathrm{~Hz}$ train for $200 \mathrm{~ms}$; pulses: $300 \mathrm{~Hz}, 150$ $\mu$ s biphasic, $50 \mu \mathrm{A}$ ), and combined electrical and optical stimulation (dark blue background). Responses were averaged in a small ROI close to the electrode tip (shown in c). Scale bar, $5 \mathrm{~mm}$. $\boldsymbol{b}$, fMRI peristimulus time course (aligned to conditions onsets). $c$, Coronal section aligned to stereotactic coordinates ( $26 \mathrm{~mm}$ anteroposterior) showing significant activity during electrical stimulation blocks (left), combined stimulation (middle), and optical stimulation alone (right).

saccades. Only sites in which reliable saccades could be evoked by electrical microstimulation and units modulated by light were investigated.

An example experiment is presented in Figure $6 a$ (data from monkey J). In this case, inhibition had no significant effect on the number of evoked saccades during combined stimulation compared with electrical stimulation alone. Although we observed a small number of saccades in the optical stimulation alone condition, those were not oriented toward a consistent direction and could be considered random eye movements that happened to align with stimulation onset (Fig. $6 a$, bottom row). We also observed cases in which combined electrical and optical stimulation resulted in a significant reduction in the number of evoked saccades (Fig. 6b, data from monkey B) compared with the number of saccades evoked with electrical stimulation alone. Although we observed a small number of saccades after the optical stimulation ( $n=12$ of 203), they were not oriented toward a consistent direction (Fig. 6b, bottom row).

Overall, of 72 stimulation experiments (in 10 sites of two monkeys; Table 2; see population average in Fig. $6 c$ ), only 12 were found to significantly modulate the number of evoked saccades (Fig. $6 d, p<0.01$, binomial test). However, the overall population was not significantly modulated (Fig. $6 d, p=0.69$, paired two-tailed $t$ test), and some of the significant experiments led to a decrease whereas some led to an increase in saccade probability.
No significant change in saccade direction was found during combined electrical and optical inhibition compared with electrical stimulation alone (Fig. $6 e, p=0.05$, paired two-tailed $t$ test), and only one experiment led to a significant increase in saccade amplitude. Saccade latencies were also not statistically different ( $p=0.23$, Kolmogorov-Smirnov test; Fig. $6 f)$. These results suggest that local inhibition of the FEF does not lead to saccade initiation and that local inhibition during electrical stimulation in most cases is not strong enough to overcome the electrical stimulation.

\section{fMRI activity during electrical and optical stimulation in} the FEF

One explanation proposed previously to explain the lack of evoked movements after optogenetic stimulation in the macaque is that the volume of stimulated tissue is much smaller compared with that evoked with electrical stimulation (Han et al., 2009; Diester et al., 2011). Previous studies suggested that optogenetics can be combined with fMRI to measure the evoked activity across the brain (Lee et al., 2010; Desai et al., 2011; Gerits et al., 2012) but have not directly compared how the pattern of activation induced by electrical stimulating differs from that induced by optical stimulation. Here, we measured fMRI activity while simultaneously applying electrical, 

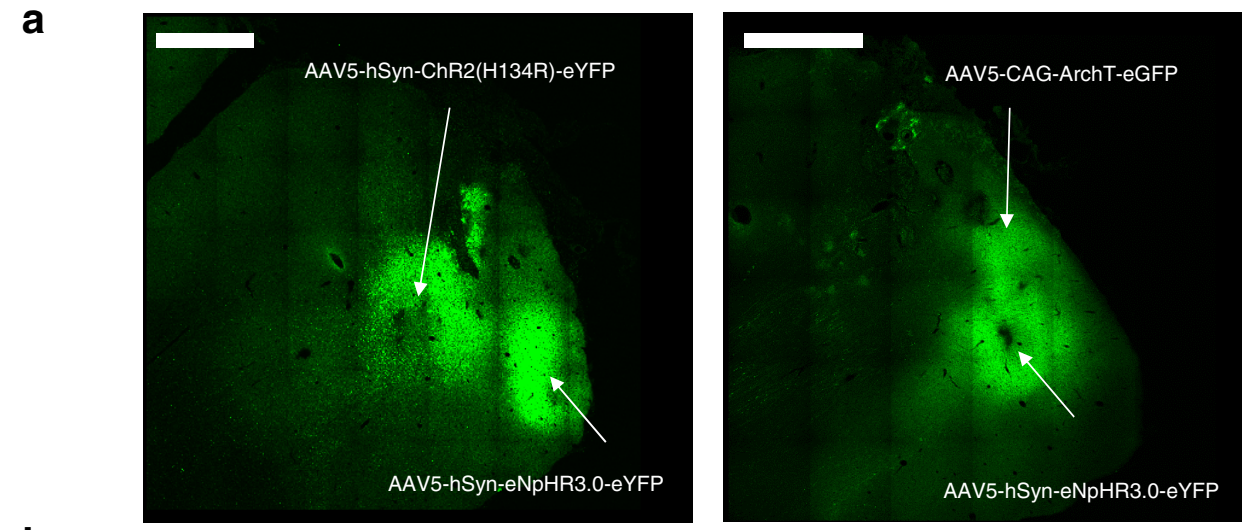

b
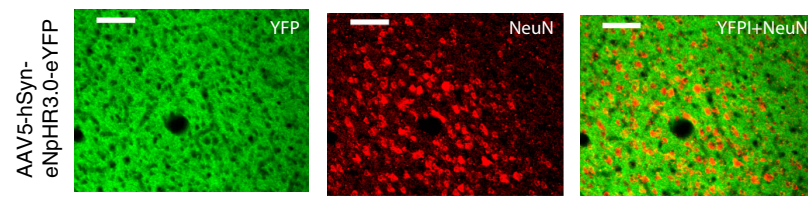

c

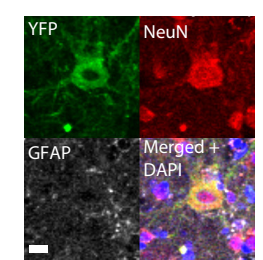

AAV5-hSyn-eNpHR3.0-eYFP

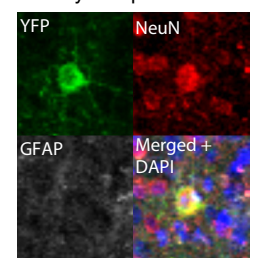

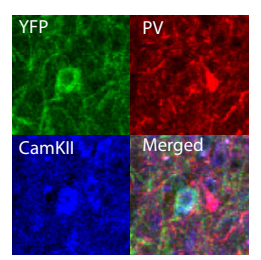

AAV5-CAG-ArchT-eGFP

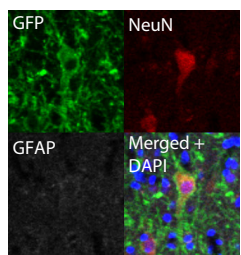

d

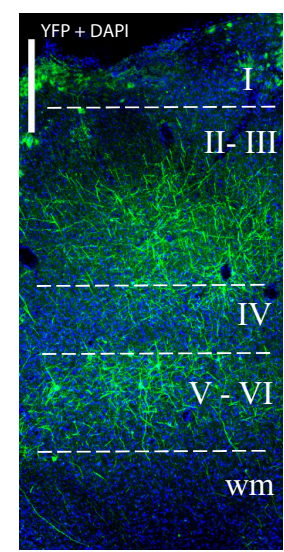

Figure 8. Injection sites and immunohistochemistry in monkey B. $\boldsymbol{a}$, Wide-field fluorescence. Scale bars, $2 \mathrm{~mm}$. $\boldsymbol{b}$, Expression close to the injection site. Left, YFP; middle, NeuN; right, YFP and NeuN. Scale bars, $500 \mu \mathrm{m}$. c, Immunohistochemistry of four example cells taken from ChR2, Arch, and eNphR3.0 sites. Opsin fluorophore (YFP/GFP, green), pan-neuronal maker (NeuN, red), cell nucleus (DAPI, blue), astroglia (GFAP, white), and excitatory marker (CaMKII, blue). Scale bar, $10 \mu \mathrm{m}$. d, YFP expression pattern across cortical layers (DAPl in blue).

optical, or combined stimulation to the FEF to assess the spread of activity.

An optrode was lowered to FEF until saccades could be evoked with low current electrical microstimulation $(<50 \mu \mathrm{A})$ and single units were modulated by light. The monkey was transported to the scanner and scanned while it performed a fixation task. We delivered pulses of optical stimulation, electrical stimulation, or both in a block design to directly assess the contribution of each type of stimulation (Fig. 7a).

The average time course of a small ROI centered close to the optrode tip is shown in Figure 7a. We found that the fMRI signal increased significantly during electrical stimulation and also during combined stimulation but not during optical stimulation alone (Fig. $7 b, c)$. The smallest $p$ value in the ROI was above 0.01 for the contrast Optical-Rest. This result was reproducible across multiple days in different sites, with different configurations: various optical stimulation trains, thicker optical fibers, optic fibers with higher numerical aperature, and two-optrode simultaneous stimulation in neighboring sites. Furthermore, no activation during optical stimulation was observed in monkey B's special site in which optical stimulation did evoke saccades (Fig. $7 a$, top).

\section{Histological analyses}

At the end of the experimental period, monkey B was perfused and standard immunohistochemistry procedures were per- formed to validate opsin expression. We observed strong expression at all three injection sites (wide-field fluorescence; Fig. $8 a, b$, no antibodies were used to amplify YFP). We labeled cells with NeuN (pan-neuronal marker), GFAP (astrocytes marker), CaMKII (excitatory maker), and PV (inhibitory marker). Most YFP-expressing cells were found within $2 \mathrm{~mm}$ of the injection site (several examples in Fig. 8c) and expressed NeuN as well. No expression was found in GFAP-positive cells. We counted $\sim 100$ cells that expressed CaMKII and YFP, but only one cell was found to express YFP and PV (PV is known to label only a subset of inhibitory neurons, which may explain the low count number). We observed YFP expression mainly in layers II-III and V-VI (assessed from DAPI staining; Fig. $8 d$ ). Tissue damage was observed in superficial regions from guide tube penetration as well as in deep tissue from repetitive optrode insertion.

To analyze the extent of the viral expression quantitatively and to assess whether there was a significant increase in opsin expression at the unique ChR2 site in which optical stimulation evoked saccades, a custom software was developed to register images across different modalities (MRI, frozen tissue block, wide-field fluorescence, high-magnification $Z$-stacks; Fig. 9a). This enabled us to represent labeled cells relative to the MRI and the nonvertical injection penetration track (see injection reconstruction in Fig. $9 b$; note that depth along the penetration does not necessarily correspond to increasing cortical layers). 
a

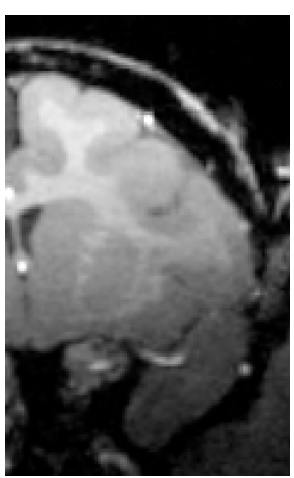

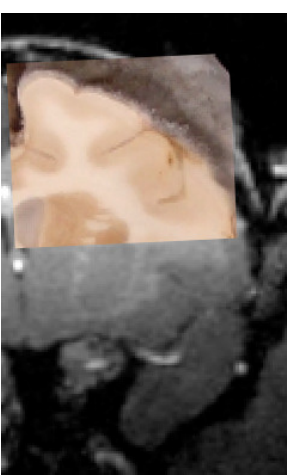

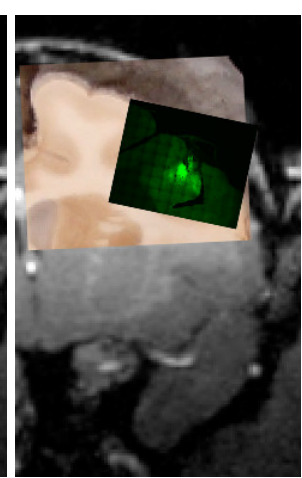

b

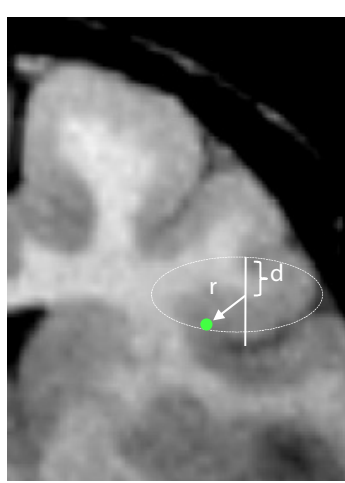

C

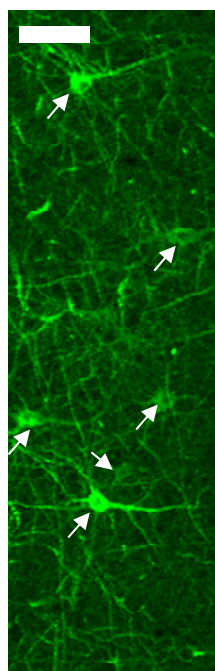

d
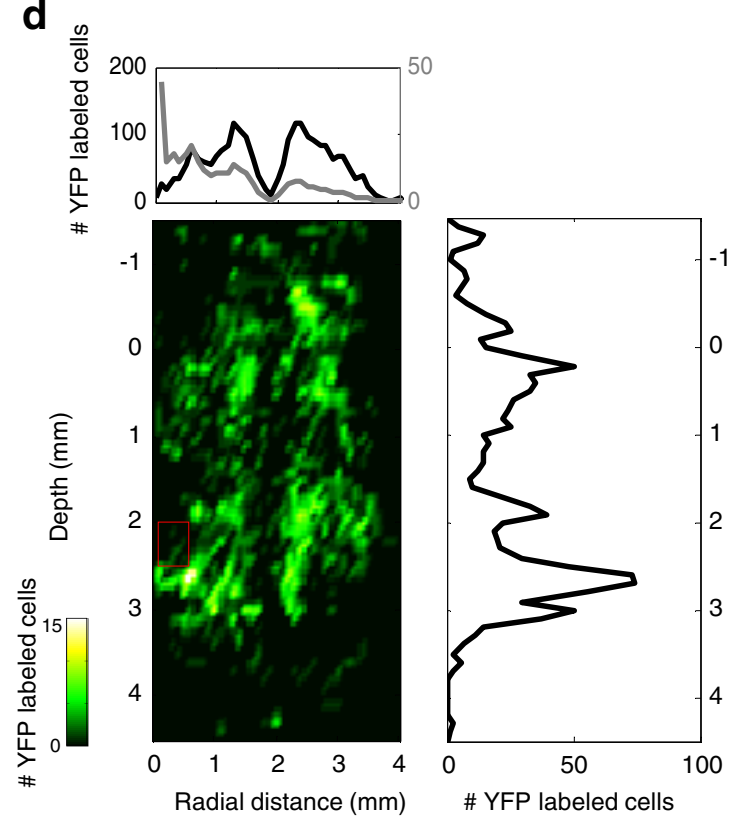

Figure 9. Quantitative histology of ChR2-expressing cells in monkey B. $\boldsymbol{a}$, Coronal MRI section of the right hemisphere showing a section of the FEF (left; scale bar, $3.5 \mathrm{~mm}$ ). The same coronal slice overlaid with a registered photograph of the frozen tissue block (middle) and the same slice overlaid with registered wide-field YFP fluorescence (right). $\boldsymbol{b}$, Schematic of the coordinate system used to represent the location of YFP-expressing cells. Injection trajectory (white line), overlaid on the reconstructed MRI scan. The YFP-expressing cell (green dot) is represented in 3D cylindrical coordinates as a function of depth (d; relative to the first injection site), radial distance (r), and angle. $\boldsymbol{c}$, Example of annotated cells in a small patch of (hR2-expressing tissue; scale bar, $20 \mu \mathrm{m}$. $\boldsymbol{d}$, Histogram of YFP-expressing cells as a function of radial distance from the injection trajectory and depth (relative to first injection site) (i.e., the cylinder was collapsed across angles to produce a $2 D$ visualization). The special site in which optical stimulation evoked saccades is highlighted in red. Top, Marginal distribution of YFP-expressing cells as a function of radial distance (black) and the distribution normalized to the radial circumference (gray). Right, Marginal distribution as a function of depth (at radial distance smaller than $2 \mathrm{~mm}$ ).

We manually annotated 3143 YFP-expressing cells (small sample tissue shown in Fig. 9c). The coordinate of each annotated cell (represented schematically as a green dot in Fig. 9b) was represented in 3D cylindrical coordinates relative to the first injection site (Fig. $9 b$, white line). A histogram of YFP-expressing cells, relative to the 3D injection path, as a function of depth and radial distance is shown in Figure $9 d$. The marginal distribution as a function of radial distance was bimodal and contained two peaks at 1.3 and $2.4 \mathrm{~mm}$. We attributed the first peak to the ChR2 injection and the second peak to the neighboring ArchT and eNpHR3.0 injections. The two peaks were separated at a valley that reached its minimum at $1.9 \mathrm{~mm}$ (this was also true when the distribution was normalized by considering the increase in circumference; Fig. $9 d$, gray curve). Therefore, we plotted the marginal distribution of ChR2 as a function of depth for only YFP-expressing cells within $2 \mathrm{~mm}$ radius (Fig. $9 d$, right plot). We found that the peak expression levels were at a depth of $2.7 \mathrm{~mm}$. No significant increase in YFP-labeled cells was found between 2 and $2.5 \mathrm{~mm}$, corresponding to the special site in which saccades were observed with optical stimulation ( $p>0.5$, bootstrapping; Fig. $9 d$, red box).

\section{Discussion}

In this study, we injected several optogenetic constructs into the macaque FEF to examine the effects of optical stimulation in evoking saccadic eye movements. On average, $74 \%$ of the single units recorded in injected regions significantly modulated their firing rate during optical stimulation. This number is slightly higher than previously reported numbers $(40-50 \%)$ (Diester et al., 2011) and may be related to the larger volumes we injected (with similar titers) or to larger area covered with lowered electrode impedance.

Recently, three studies emerged with reports about optogenetic manipulation in macaques leading to observed behavioral changes. Gerits et al. (2012) reported a reduction in response latencies when monkeys performed a visually guided saccade task during ChR2 stimulation in the FEF but no optically evoked saccades. Cavanaugh et al. (2012) found that inactivation of the 
superior colliculus leads to a small shift in saccade endpoint and a slight increase in saccade latency. Jazayeri et al. (2012) trained animals to respond to flashes of light with an eye movement and reported consistent saccades to the same location after ChR2 stimulation in primary visual cortex, suggesting that stimulation evoked the perception of a phosphene. Although these studies are important in providing evidence that optogenetic perturbations can lead to behavioral changes, they do not address the issue of how effective optogenetic perturbations are compared with the most widely used method of perturbing behavior in monkeys, electrical stimulation. They also do not address how optogenetic and electrical perturbations interact, a question important for clarifying whether the two types of perturbations act on common circuits.

By examining the effects of optogenetic constructs side by side with electrical microstimulation, we show that ChR2 optical stimulation has an effect similar to low current (or low frequency) electrical stimulation. In ChR2-injected regions, optical stimulation did not evoke a saccade in most cases, despite strong neural modulation. The question whether these neural perturbations contribute in any way to initiation of a saccade was answered by coupling the optical stimulation to low current electrical stimulation. A significant increase in the number of evoked saccades was observed, essentially increasing the efficacy of electrical stimulation, suggesting that optical stimulation contributes to network activity that can evoke a movement, but without the low electrical current injection, the effects remain subthreshold and are not strong enough to initiate a movement. In particular, when electrical current was so low such that it alone did not evoke any saccades, the addition of optical stimulation did lead to evoked saccades. This result rules out previously the hypothesized idea that optical stimulation modulates cells that do not participate in generating behavior or that stimulation activates neurons not in the right way needed to generate a movement (Diester et al., 2011). Our results suggest that optical and electrical stimulation work on a functionally overlapping population of cells because the behavioral effects are additive.

In one monkey, we found that optical stimulation alone reliably evoked saccades. Modulating the optical stimulation train parameters revealed that higher stimulation frequencies were more effective in driving saccades and that movements could be evoked with as little as $82 \mathrm{~mW} / \mathrm{mm}^{2}$. This suggested that ChR2 with improved channel kinetics that allow higher stimulation frequencies may be more effective in driving behavior. However, optical stimulation in a third monkey injected with secondgeneration ChR2 (Gunaydin et al., 2010; Mattis et al., 2012) did not evoke saccades, nor did a construct targeting pyramidal neurons [CaMKII-ChR2(E123A)]. It is important to note that neurons in both regions injected with these two variants showed strong modulation (similar number of modulated cells to those observed in the two other monkeys) and that an increase in saccade probability was observed when optical stimulation was coupled to low current electrical stimulation. Thus, second-generation ChR2, although capable of driving cells to higher frequencies, failed to evoke a movement in the tested sites (we do not rule out the possibility that ChETA (ChR2E123T accelerated) variants may work in sites that may have some special characteristics, like the one we observed in monkey B).

The level of electrical current needed to evoke saccades did not differ significantly in the special site of monkey B compared with nearby sites in which optical stimulation was not effective, sug- gesting that it was not attributable to some basic physiological difference, such as a lower spike threshold. We propose two plausible explanations why saccades were evoked in this site. One is that the site included a larger fraction of cells encoding the same saccade direction, rendering focal optical stimulation more effective. This was supported by our analysis showing that electrically evoked saccades in that region were the most accurate ones. Another possibility is that the region had a significant increase in viral expression. Our histological analysis did not show a significant increase in expression at the special site in which saccades were evoked with optical stimulation. However, we did find a small peak of YFP-expressing cells at a depth of $2.7 \mathrm{~mm}$ and cannot rule out completely that the mismatch is attributable to small errors caused by nonlinear tissue deformation (e.g., caused by repetitive fiber insertion in the following experiments).

Previous studies have shown that combined fMRI and electrical stimulation can reveal regions connected to the site of stimulation (Tolias et al., 2005; Moeller et al., 2008; Logothetis et al., 2010). Experiments with rodents using combined fMRI and optical stimulation suggested that ChR2 stimulation can be used to trace anatomical connections (Lee et al., 2010; Desai et al., 2011). In contrast to a recent report by Gerits et al. (2012) who stimulated NHPs with ChR2 in FEFs, we found no significant BOLD activation elicited by ChR2 stimulation close to the optrode tip (but strong activation during interleaved electrical stimulation blocks). We made numerous attempts to replicate the result of Gertis et al., including using various second-generation ChR2 constructs, different stimulation parameters, thicker fibers diameters, and simultaneously stimulating two adjacent sites. Thus, one possible explanation why Gertis et al. observed significant activation might be related to their use of an active saccade task in the scanner compared with the fixation paradigm used in this study.

The correlation between the large behavioral response evoked by electrical stimulation and the strong fMRI activity observed at the stimulation site during electrical stimulation suggests that a critical difference between electrical and optogenetic stimulation may be the volume of stimulated tissue. However, optical stimulation at the effective site in monkey B (Fig. 7), which evoked saccades, did not lead to a significant BOLD increase, suggesting that motor movements can be evoked with small stimulated volumes (that may not be visible at $1 \mathrm{~mm}^{3} \mathrm{fMRI}$ scanning resolution).

Low current electrical stimulation in the FEF is sufficient to evoke saccades and induce strong fMRI activity close to the tip and presumably activates a distributed sparse network of cells (Histed et al., 2009). It is possible that that these cells are highly functionally correlated, and optogenetic stimulation fails to stimulate similarly functionally homogeneous networks. If this is the case, increasing the reliability of optogenetic stimulation in evoking motor movements may not necessarily require engineering tools with larger expression volumes or light that penetrates deeper (e.g., C1V1; Yizhar et al. 2011a) but rather targeting sparse functional correlated networks.

Although optogenetics has many advantages over electrical stimulation, our experiments suggest that the classical method of evoking motor movement with direct current injection is still more effective compared with existing optogenetic constructs. Optical stimulation led in most cases to a subthreshold activity that was not sufficient to initiate a movement. However, such perturbation can be used to modulate ongoing motor behavior, such as latency or direction (Cavanaugh et al., 2012; Gerits et al., 2012). Optogenetics has revolutionized neuroscience, making it 
possible to test hypotheses about how local circuit perturbations affect or generate behavior. Our demonstration that ChR2 stimulation increases the efficacy of low current electrical stimulation and that ChR2 stimulation alone can evoke saccades is an important step in establishing the applicability of the technique for dissecting behaviorally relevant neural circuits in NHPs and paves the way for additional investigation to discover the necessary and sufficient conditions required for evoking motor responses.

\section{References}

Berens P (2009) CircStat: a Matlab toolbox for circular statistics. J Stat Software 31. http://www.jstatsoft.org/v31/i10.

Boyden ES, Zhang F, Bamberg E, Nagel G, Deisseroth K (2005) Millisecondtimescale, genetically targeted optical control of neural activity. Nat Neurosci 8:1263-1268. CrossRef Medline

Bruce CJ, Goldberg ME, Bushnell MC, Stanton GB (1985) Primate frontal eye fields. II. Physiological and anatomical correlates of electrically evoked eye movements. J Neurophysiol 54:714-734. Medline

Cavanaugh J, Monosov IE, McAlonan K, Berman R, Smith MK, Cao V, Wang KH, Boyden ES, Wurtz RH (2012) Optogenetic inactivation modifies monkey visuomotor behavior. Neuron 76:901-907. CrossRef Medline

Chow BY, Han X, Dobry AS, Qian X, Chuong AS, Li M, Henninger MA, Belfort GM, Lin Y, Monahan PE, Boyden ES (2010) High-performance genetically targetable optical neural silencing by light-driven proton pumps. Nature 463:98-102. CrossRef Medline

Cohen MR, Newsome WT (2004) What electrical microstimulation has revealed about the neural basis of cognition. Curr Opin Neurobiol 14:169177. CrossRef Medline

Desai M, Kahn I, Knoblich U, Bernstein J, Atallah H, Yang A, Kopell N, Buckner RL, Graybiel AM, Moore CI, Boyden ES (2011) Mapping brain networks in awake mice using combined optical neural control and fMRI. J Neurophysiol 105:1393-1405. CrossRef Medline

Diester I, Kaufman MT, Mogri M, Pashaie R, Goo W, Yizhar O, Ramakrishnan C, Deisseroth K, Shenoy KV (2011) An optogenetic toolbox designed for primates. Nat Neurosci 14:387-397. CrossRef Medline

Ekstrom LB, Roelfsema PR, Arsenault JT, Bonmassar G, Vanduffel W (2008) Bottom-up dependent gating of frontal signals in early visual cortex. Science 321:414-417. CrossRef Medline

Galvan A, Hu X, Smith Y, Wichmann T (2012) In vivo optogenetic control of striatal and thalamic neurons in non-human primates. PLoS One 7:e50808. CrossRef Medline

Gerits A, Farivar R, Rosen BR, Wald LL, Boyden ES, Vanduffel W (2012) Optogenetically induced behavioral and functional network changes in primates. Curr Biol 22:1722-1726. CrossRef Medline

Goldberg ME, Bushnell MC, Bruce CJ (1986) The effect of attentive fixation on eye movements evoked by electrical stimulation of the frontal eye fields. Exp Brain Res 61:579-584. Medline

Gradinaru V, Thompson KR, Zhang F, Mogri M, Kay K, Schneider MB, Deisseroth K (2007) Targeting and readout strategies for fast optical neural control in vitro and in vivo. J Neurosci 27:14231-14238. CrossRef Medline

Gradinaru V, Thompson KR, Deisseroth K (2008) eNpHR: a Natronomonas halorhodopsin enhanced for optogenetic applications. Brain Cell Biol 36:129-139. CrossRef Medline

Gradinaru V, Zhang F, Ramakrishnan C, Mattis J, Prakash R, Diester I, Goshen I, Thompson KR, Deisseroth K (2010) Molecular and cellular approaches for diversifying and extending optogenetics. Cell 141:154-165. CrossRef Medline

Graziano MS, Taylor CS, Moore T (2002) Complex movements evoked by microstimulation of precentral cortex. Neuron 34:841-851. CrossRef Medline

Gunaydin LA, Yizhar O, Berndt A, Sohal VS, Deisseroth K, Hegemann P
(2010) Ultrafast optogenetic control. Nat Neurosci 13:387-392. CrossRef Medline

Han X (2012) Optogenetics in the nonhuman primate. Prog Brain Res 196: 215-233. CrossRef Medline

Han X, Qian X, Bernstein JG, Zhou HH, Franzesi GT, Stern P, Bronson RT, Graybiel AM, Desimone R, Boyden ES (2009) Millisecond-timescale optical control of neural dynamics in the nonhuman primate brain. Neuron 62:191-198. CrossRef Medline

Han X, Chow BY, Zhou H, Klapoetke NC, Chuong A, Rajimehr R, Yang A, Baratta MV, Winkle J, Desimone R, Boyden ES (2011) A high-light sensitivity optical neural silencer: development and application to optogenetic control of non-human primate cortex. Front Syst Neurosci 5:18. CrossRef Medline

Harris KD, Henze DA, Csicsvari J, Hirase H, Buzsáki G (2000) Accuracy of tetrode spike separation as determined by simultaneous intracellular and extracellular measurements. J Neurophysiol 84:401-414. Medline

Histed MH, Bonin V, Reid RC (2009) Direct activation of sparse, distributed populations of cortical neurons by electrical microstimulation. Neuron 63:508-522. CrossRef Medline

Jazayeri M, Lindbloom-Brown Z, Horwitz GD (2012) Saccadic eye movements evoked by optogenetic activation of primate V1. Nat Neurosci 15:1368-1370. CrossRef Medline

Lee JH, Durand R, Gradinaru V, Zhang F, Goshen I, Kim DS, Fenno LE, Ramakrishnan C, Deisseroth K (2010) Global and local fMRI signals driven by neurons defined optogenetically by type and wiring. Nature 465:788-792. CrossRef Medline

Logothetis NK, Augath M, Murayama Y, Rauch A, Sultan F, Goense J, Oeltermann A, Merkle H (2010) The effects of electrical microstimulation on cortical signal propagation. Nat Neurosci 13:1283-1291. CrossRef Medline

Mattis J, Tye KM, Ferenczi EA, Ramakrishnan C, O'Shea DJ, Prakash R, Gunaydin LA, Hyun M, Fenno LE, Gradinaru V, Yizhar O, Deisseroth K (2012) Principles for applying optogenetic tools derived from direct comparative analysis of microbial opsins. Nat Methods 9:159-172. CrossRef Medline

Moeller S, Freiwald WA, Tsao DY (2008) Patches with links: a unified system for processing faces in the macaque temporal lobe. Science 320:13551359. CrossRef Medline

Moore T, Fallah M (2004) Microstimulation of the frontal eye field and its effects on covert spatial attention. J Neurophysiol 91:152-162. CrossRef Medline

Murphey DK, Maunsell JH (2007) Behavioral detection of electrical microstimulation in different cortical visual areas. Curr Biol 17:862-867. CrossRef Medline

Ohayon S, Tsao DY (2012) MR-guided stereotactic navigation. J Neurosci Methods 204:389-397. CrossRef Medline

Ruiz O, Lustig BR, Nassi JJ, Cetin A, Reynolds JH, Albright TD, Callaway EM, Stoner GR, Roe AW (2013) Optogenetics through windows on the brain in the nonhuman primate. J Neurophysiol 110:1455-1467. CrossRef Medline

Salzman CD, Britten KH, Newsome WT (1990) Cortical microstimulation influences perceptual judgements of motion direction. Nature 346:174177. CrossRef Medline

Tolias AS, Sultan F, Augath M, Oeltermann A, Tehovnik EJ, Schiller PH, Logothetis NK (2005) Mapping cortical activity elicited with electrical microstimulation using FMRI in the macaque. Neuron 48:901-911. CrossRef Medline

Yizhar O, Fenno LE, Prigge M, Schneider F, Davidson TJ, O'Shea DJ, Sohal VS, Goshen I, Finkelstein J, Paz JT, Stehfest K, Fudim R, Ramakrishnan C, Huguenard JR, Hegemann P, Deisseroth K (2011a) Neocortical excitation/inhibition balance in information processing and social dysfunction. Nature 477:171-178. CrossRef Medline

Yizhar O, Fenno LE, Davidson TJ, Mogri M, Deisseroth K (2011b) Optogenetics in neural systems. Neuron 71:9-34. CrossRef Medline 